\title{
Modular Control of Pointing beyond Arm's Length
}

\author{
Bastien Berret, ${ }^{1,2}$ François Bonnetblanc, ${ }^{1,2}$ Charalambos Papaxanthis, ${ }^{1,2}$ and Thierry Pozzo ${ }^{1,2,3}$ \\ ${ }^{1}$ Université de Bourgogne, Dijon, Campus Universitaire, Unité de Formation et de Recherche en Sciences et Techniques des Activités Physiques et Sportives, \\ and 'Institut National de la Santé et de la Recherche Médicale, Unité 887, Motricité-Plasticité, F-21078 Dijon, France, and 3'Italian Institute of Technology, \\ 16163 Genoa, Italy
}

Hand reaching and bipedal equilibrium are two important functions of the human motor behavior. However, how the brain plans goal-oriented actions combining target reaching with equilibrium regulation is not yet clearly understood. An important question is whether postural control and reaching are integrated in one single module or controlled separately. Here, we show that postural control and reaching motor commands are processed by means of a modular and flexible organization. Principal component and correlation analyses between pairs of angles were used to extract global and local coupling during a whole-body pointing beyond arm's length. A low-dimensional organization of the redundant kinematic chain allowing simultaneous target reaching and regulation of the center of mass (CoM) displacement in extrinsic space emerged from the first analysis. In follow-up experiments, both the CoM and finger trajectories were constrained by asking participants to reach from a reduced base of support with or without knee flexion, or by moving the endpoint along a predefined trajectory (straight or semicircular trajectories). Whereas joint covaried during free conditions and under equilibrium restrictions, it was decomposed in two task-dependent and task-independent modules, corresponding to a dissociation of arm versus legs, trunk, and head coordination, respectively, under imposed finger path conditions. A numerical simulation supported the idea that both postural and focal subtasks are basically integrated into the same motor command and that the CNS is able to combine or to separate the movement into autonomous functional synergies according to the task requirements.

Key words: whole-body pointing; equilibrium; center of mass; finger trajectory; joint coupling; modular control

\section{Introduction}

Most of our daily life activities involve the entire body. However, the two traditional ways to investigate human motor control either focus on the equilibrium mechanisms (for review, see Horak and McPherson, 1996) without considering hand trajectory formation or, conversely, on reaching movements by artificially restricting the motion of the lower body parts (for review, see Shadmehr and Wise, 2005). Nevertheless, the picture of how the CNS plans goal-oriented action may probably be clarified by asking how all body parts are coordinated during a pointing task. For instance, when reaching beyond arm's length from a standing posture, the trunk-plus-arm action causes a displacement of the whole body with respect to the direction of gravity. Thus, in addition to arm trajectory formation and target reaching, the CNS must anticipate the potential loss of balance.

An open question is whether these two subtasks are controlled as one single central command or separately (Hess, 1954; Belenkii et al., 1967; Cordo and Nashner, 1982; Schepens and Drew, 2003). A classical approach is to consider the two mechanisms in a hierarchical manner: equilibrium maintenance would be subordinated to reaching and lower limbs would offset the displace-

Received July 22, 2008; revised Nov. 24, 2008; accepted Nov. 26, 2008.

This work was supported by Centre National d'Etudes Spatiales and by Conseil Régional de Bourgogne. We thank C. Darlot, P. Morasso, and P. J. Stapley for their useful comments.

Correspondence should be addressed to Thierry Pozzo, Institut National de la Santé et de la Recherche Médicale, Unité887, Campus Universitaire, Motricité-Plasticité,F-21078 Dijon, France. E-mail:thierry.pozzo@u-bourgogne.fr. DOI:10.1523/JNEUROSCI.3426-08.2009

Copyright $\odot 2009$ Society for Neuroscience $\quad$ 0270-6474/09/290191-15\$15.00/0 ment of the center of mass (CoM) accompanying voluntary arm movement (Massion, 1992). According to this interpretation, lower and upper body parts would ensure equilibrium preservation and finger trajectory formation, respectively. Alternatively, a grouping of body angles was proposed as a kinematic solution to reduce the effective number of degrees of freedom and simplify the motor command (Bernstein, 1967; Alexandrov et al., 1998, 2001a,b; Lacquaniti et al., 1999; Thomas et al., 2005). In this case, equilibrium and reaching subtasks would be integrated in the same module, a term that we use to denote the covariation of a specific set of angles. To check whether the CNS would control one or several functional modules regrouping or dissociating focal and equilibrium task objectives, we tested the hypothesis that, according to the task demand, the CNS controls the whole body either as one single or two distinct modules. The finding of both global and local kinematic synergies would support a flexible modular organization (Bizzi et al., 2008) able to integrate or dissociate, if necessary, equilibrium maintenance and target reaching rather than a subordinate mode of control.

Our approach consisted of applying equilibrium and spatial constraints on a natural unstable hand reaching and analyze the kinematics of the resulting movements to infer the mechanisms underlying the coordination of the two subtasks. In one condition, reaching was performed from a reduced base of support or without knee flexion to limit the capacity of producing horizontal or vertical CoM displacements. In another condition, subjects were asked to reach the target following a straight or semicircular finger trajectory. The priority given to one of the subtasks may clarify how the different body parts enter into coordination. Fig- 
ure 1 sums up the possible motor strategies in response to these experimental restrictions. Reaching a target under postural or focal constraints could produce a global effect in which all body parts are integrated into one module, supporting the idea of a synergic control of postural and goaloriented aspects of the action. Alternatively, the motor planning could be split in two distinct modules, one driving arm reaching and one maintaining equilibrium; the two modules could be new or be derived from the basic motor strategy initially present in the free condition. The results show that, when priority is given to hand trajectory, a second module originates from the basic kinematic strategy suggesting a flexible coordination of posture and movement.

\section{Materials and Methods}

Participants. Twenty-six adults (all males, $18-29$ years; mean height, $1.76 \pm 0.08 \mathrm{~m}$; mean weight, $68 \pm 9 \mathrm{~kg}$ ) agreed voluntarily to participate in the experiments. All were healthy with no previous neurological disease and normal or corrected-to-normal vision. The experiments conformed to the Declaration of Helsinki and informed consent was obtained from all the participants according to the protocol of the Ethics Committee of the Université de Bourgogne. The experimental protocol is illustrated in Figure 2. The participants were divided in two groups: the first one performed the "basic" and "equilibrium" experimental tasks (Fig. 2a,b, b'), whereas the second group performed the "basic" and "spatial" experimental tasks (Fig. 2a,c, $c^{\prime}$ ).

Basic motor task. All the participants performed the basic motor task ( $\mathrm{B}$ condition) (Fig. 2a) and the recorded results served as reference to compare the results of the other experimental tasks. A similar protocol was already used in recent studies (Pozzo et al., 2002; Schmid et al., 2006). Participants were asked to point with the two arms simultaneously toward a pair of targets placed at two different distances [lateral coordinates: $Z= \pm 0.2 \mathrm{~m}$; vertical coordinates: $Y=15 \%$ of each participant's height; anteroposterior (A-P) coordinates: $X$, depending on the target distance]. The first target distance corresponded to $5 \%$ (short distance, D1), and the second to $30 \%$ (long distance, D2) of the participants' height. Both distances were measured from the distal end of the participants' great toe. Participants started from an upright standing position and their hands were located initially at the external side of the thighs and thus induced hand-pointing movements in a semipronated position. The whole movement was performed in the sagittal plane, with each side of the body moving together. Target accuracy was not the primary constraint imposed on the participants during the experiments, and no instruction was given to them regarding the movements to be effected by the body segments. Each participant achieved, at a natural (self-selected) speed, a block of 10 pointing movements for each condition of distance.

Equilibrium constraint. Twelve participants took part in the experiment in which equilibrium constraints were added to the basic motor task (Fig. $2 b, b^{\prime}$ ). Equilibrium constraints were introduced in two different ways. Participants were asked to point toward the targets (1) by freezing their knee joints, a condition that imposed a high inverted pendulum configuration reducing stability (K condition, extended knee condition) (Fig. 2b), and (2) from a reduced base of support (R condition) defined by a $40 \mathrm{~cm}$ large horizontal square (wooden board) fixed on a thin piece of wood ( $5 \mathrm{~cm}$ high, $5 \mathrm{~cm}$ large, and $40 \mathrm{~cm}$ long) (Fig. $\left.2 b^{\prime}\right)$. In both conditions, they were asked to perform the motor task without falling from the small support or loosing equilibrium control. Participants stayed on the wooden board with the vertical projection of the malleolus of each foot at the backward limit of the thin piece of wood. Both motor tasks (K and R) were executed at a natural pace. Within each motor task, the number of trials and the order of execution of the two distances were similar to those of the basic motor task. One or two preliminary trials allowed participants to familiarize themselves with the experimental restrictions.

Spatial constraint. Fourteen participants took part in the experiment (Fig. $2 c, c^{\prime}$ ) in which spatial constraints were added to the basic motor task. Participants were asked to point to the targets by using a straight finger trajectory (S condition) (Fig. $2 c$ ). Participants initially performed three nonrecorded trials by following a straight wire connecting the initial finger position to the target. After this short period, they were asked to perform the task without wire and to keep the same speed as in previous experiments. Participants were also requested to reach the targets with large finger path curvatures ( $\mathrm{C}$ condition; semicircular finger trajectory) (Fig. $2 c^{\prime}$ ). The imposed path was concave in the sagittal plane (semicircle whose diameter was equal to the distance between the initial finger position and the target). Again, the participants performed three nonrecorded trials by tracking a curved wire connecting the initial finger position and the targets. Then, they were asked to perform the task without wire. Both tasks were performed at a natural pace. Within each motor task, the number of trials and the order of execution of the two distances were similar to those of the basic motor task. The participants were allowed to rest $1 \mathrm{~min}$ between each condition and the total duration of the whole experiment was $1.5 \mathrm{~h}$.

Data collection and processing. Movement kinematics was captured using an optoelectronic device (SMART-BTS). Nine cameras were used to measure the position of 11 retro-reflective markers ( $15 \mathrm{~mm}$ in diameter), which were placed at various anatomical locations on the right side of the body (external cantus of the eye, auditory meatus, acromial process, humeral lateral condyle, ulnar styloid process, apex of the index finger, D1 vertebra, greater trochanter, knee interstitial joint space, external malleolus, and fifth metatarsal head of the foot).

All analyses were performed with custom software written in Matlab (Mathworks) from the recorded three-dimensional position of the 11 markers (sampling frequency, $120 \mathrm{~Hz}$ ). Recorded signals were low-pass filtered using a digital fifth-order Butterworth filter at a cutoff frequency of $7.5 \mathrm{~Hz}$ (Matlab filtfilt function). The whole-body motion was per- 

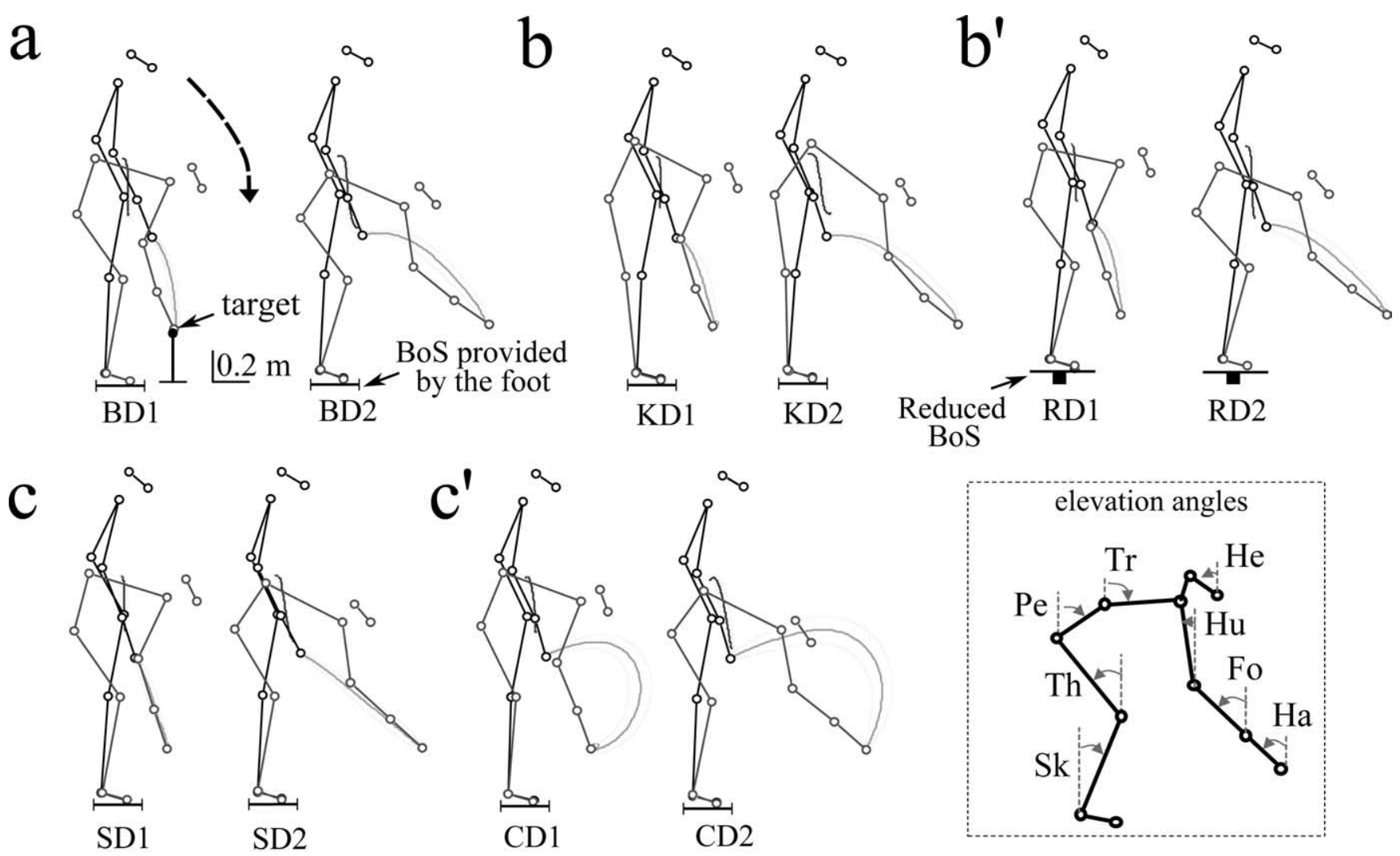

Figure 2. Stick diagrams of the task performed under basic condition ( $\boldsymbol{a}$ ), equilibrium constraints $\left(\boldsymbol{b}, \boldsymbol{b}^{\prime}\right)$, and spatial constraints $\left(\boldsymbol{c}, \boldsymbol{c}^{\prime}\right)$, for the near (D1) and the distant (D2) target. $\boldsymbol{a}$, Basic condition (BD1, BD2). $\boldsymbol{b}$, Knee-extended condition (KD1, KD2). $\boldsymbol{b}^{\prime}$, Reduce base of support condition (RD1, RD2). c, Imposed straight finger trajectory condition (SD1, SD2). $\boldsymbol{c}^{\prime}$, Imposed semicircular trajectory condition (CD1, CD2). The dark gray and light gray traces depict the $\mathrm{CoM}$ and the finger trajectories in the sagittal plane, respectively. The inset box defines the eight elevation angles under consideration (Sk, shank; Th, thigh; Pe, pelvis; Tr, trunk; He, head; Hu, humerus; Fo, forearm; Ha, hand).

formed in the sagittal plane. To verify the planarity hypothesis of the movement, we used a principal component analysis (PCA) (Jolliffe, 1986) on the whole three-dimensional dataset of each participant (grouping all markers as individual observations). The variance accounted for (VAF) by the two first principal components (PCs) was $>98 \%$ for all participants, indicating that most markers trajectories were approximately lying on one plane. In particular, this plane was nearly the one defined by the reference frame of the acquisition system $(X Y$, where $X$ was the A-P axis and $Y$ was the vertical axis). The angle between normal vectors of the planes was $<4 \pm 1.5^{\circ}$. Therefore, we projected the threedimensional data on the plane $X Y(X$, anteroposterior axis; $Y$, vertical axis) defined by our recording system.

Movement analysis. An estimation of CoM displacements was used to characterize the equilibrium performance. We estimated the position of the CoM using an eight-segment mathematical model consisting of the following rigid segments: head, trunk, thigh, shank, foot, upper arm, forearm, and hand. Using this model, the position of the CoM was calculated via standard procedures and using documented anthropometric parameters (Winter, 1990). The model used to determine the wholebody CoM position has previously been validated for similar whole-body reaching movements (Stapley et al., 1999). The final whole-body momentum (WBM) was also evaluated using an inverse pendulum model in which the whole-body mass was concentrated in its CoM. It was evaluated using the standard formula $m g x_{\mathrm{A}-\mathrm{P}}$, where $m$ was the subject mass, $g$ is the gravity acceleration $\left(\approx 9.81 \mathrm{~m} \cdot \mathrm{s}^{-2}\right)$, and $x_{\mathrm{A}-\mathrm{P}}$ was the distance between the ankle (fixed point) and the projection of the CoM on the A-P axis.

The finger kinematics was used to analyze the pointing performance. Finger movement onset time was defined as the instant at which the linear tangential velocity of the index fingertip exceeded $5 \%$ of its peak and the end of movement as the point at which the same velocity dropped below the $5 \%$ threshold. Standard kinematic parameters usually described in arm-pointing studies (Papaxanthis et al., 2005) were calculated as follows: movement duration (MD), peak velocity (PV), mean velocity (MV), relative time to peak velocity (TPV) defined as the ratio of the duration of acceleration and MD, index of finger path curvature (IPC = Dev/LD) defined as the ratio of the maximum path deviation (Dev) from a straight line connecting the initial and final finger positions [linear distance (LD)], and curvilinear distance of the finger (CD) defined by the integral over time from 0 to $\mathrm{MD}$ of the norm of the finger velocity vector (in plane $X Y$ ). To evaluate the consistency of the final finger position, we calculated the $95 \%$ confidence ellipses in plane $X Y$. Constant errors were defined as the distance between the finger position and the target in three dimensions.

Eight elevation angles (with respect to the vertical axis $Y$; Sk, shank; Th, thigh; Pe, pelvis; Tr, trunk; He, head; Hu, humerus; Fo, forearm; Ha, hand) were defined (Fig. 2, inset). Arm angles will be referred to as "upper limb" angles in opposition to the angles of leg, trunk, and head, which will be referred to as "lower limb" angles, for the sake of simplicity. The temporal window used for analyzing the angular displacement was based on the finger onset and ending times defined above. We visually verified that the finger marker was the first and the last to move compared with the other markers placed on the body. The angular time series are depicted in Figure 3. These time series were then used to compute (1) the amplitude of each angular displacement (defined as the absolute value of difference between the initial and final angle) and (2) an index of the whole-body coordination using PCA (see below).

All time series were time-normalized to 200 points by using Matlab routines of interpolation (Matlab spline function).

Principal component analysis. PCA was applied to the angular displacements. Each sample of time was considered as a single observation lying 


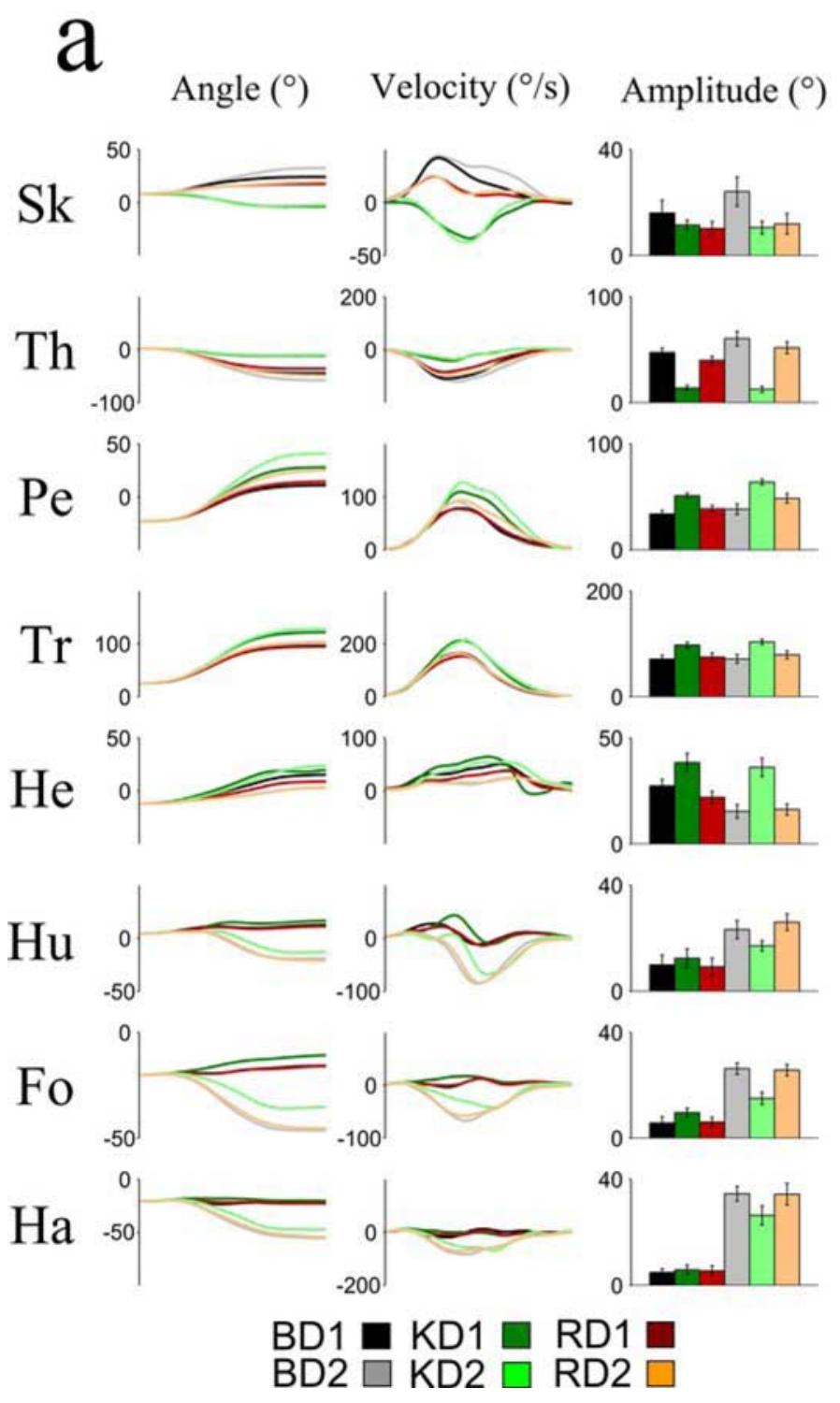

\section{$b$}

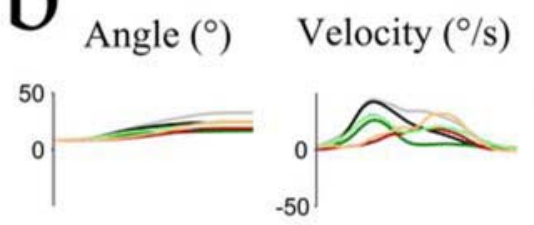

Amplitude $\left({ }^{\circ}\right)$
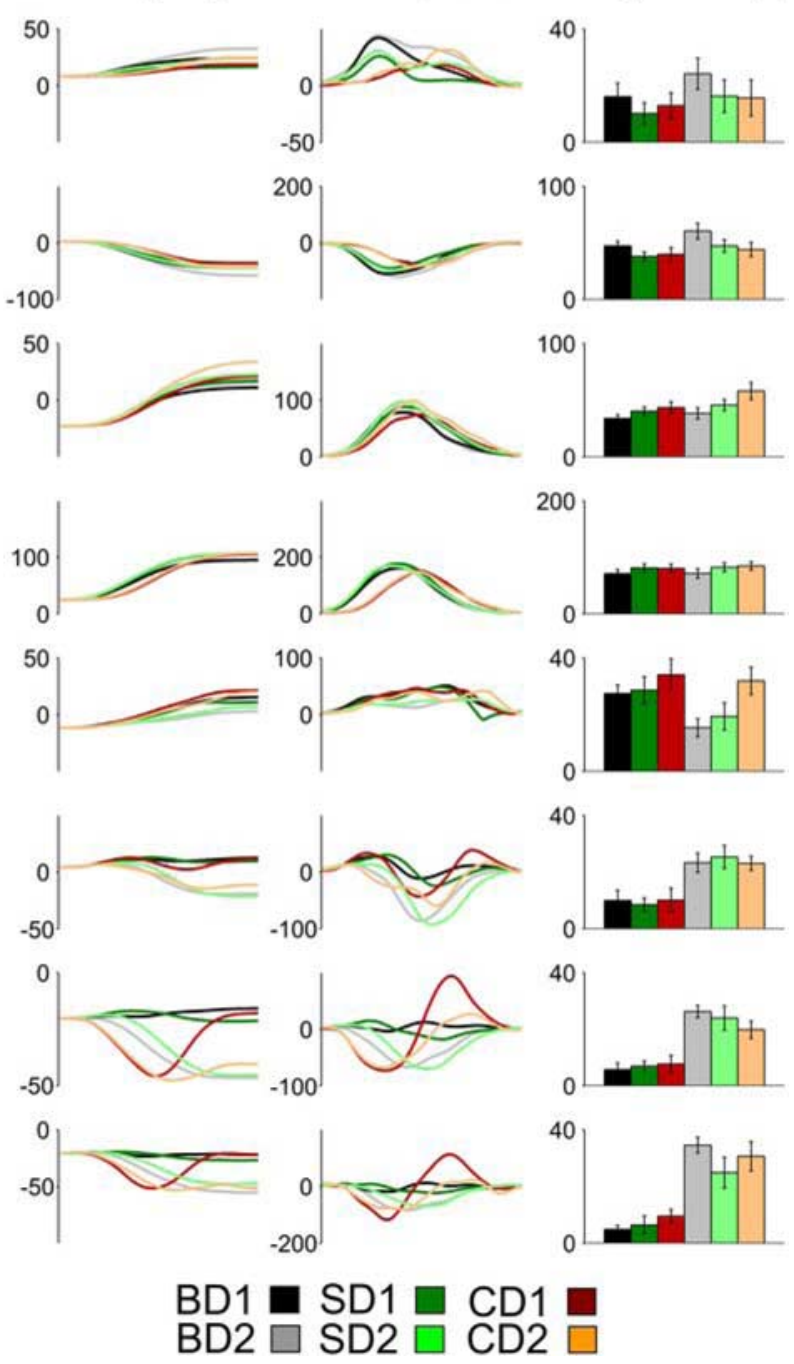

Figure 3. Angular kinematic values. $\boldsymbol{a}$, Basic (B), extended knee (K), and reduced base of support (R) conditions. From the left to the right, mean time series of the angular displacements, velocity profiles, and mean values of peak to peak angular displacements (histograms). $\boldsymbol{b}$, Same data for the basic (B), straight trajectory (S), and semicircular trajectory (C) conditions. The abbreviations are the same as in Figure 2.

in an ambient vector space whose dimension was given by the number of time series included in the analysis.

For instance, consider a simple input dataset composed of eight columns (the angular displacements recorded during one trial) and 200 rows (normalized time). PCA could be thought as a generalization of a correlation analysis in a high dimensional space. In this case, PCA extracted the commonality between the angular displacements, which was sometimes referred to as "waveforms" because it especially focused on the shape of the angular time series.

Let $\Theta$ be the matrix of time-normalized angular displacements $(200$ rows $\times 8$ columns). For each $i \epsilon\{1, \ldots, 8\}$, the $i$ th column of $\Theta$ (denoted by $\Theta_{i}$ ) was centered and normalized in amplitude using the formula $\tilde{\Theta}_{i}=$ $\left(\Theta_{i}-m_{i}\right) / s_{i}$, where $m_{i}$ and $s_{i}$ were the mean and SD of $\Theta_{i}$, respectively, and thus, we got a new matrix $\tilde{\Theta}$. Then, the covariance matrix of $\tilde{\Theta}$ was computed (i.e., the correlation matrix of $\Theta$ ) and decomposed on the basis of eigenvectors (by orthonormal diagonalization). We preferentially used the correlation matrix to take into account the different ranges of motion of each segment. After reordering the eigenvalues by decreasing order (and thus rearranging the corresponding eigenvectors), the matrix of eigenvectors was denoted by $W=\left(w_{i j}\right)_{1 \leq i, j \leq 8}$. This matrix contained the weighting coefficients, i.e., the loadings of PCA. Then, the principal components (denoted by $\Pi$, and referred to as PCs), which represented the most common waveforms contained in the input dataset, were defined as the following linear combination: $\Pi=\tilde{\Theta} W$.

For instance, the first PC (also referred to as PC1) was obtained by the following formula:

$$
\Pi_{1}=\sum_{i=1}^{i=8} w_{i 1} \tilde{\Theta}_{i}
$$

[the first column of $\Pi$ (i.e., a vector with 200 rows corresponding to the time)].

When performing PCA, it was useful to examine the loadings to uncover how angular displacements were captured by each PC. To this aim, we thus analyzed each column of $W$ (i.e., each eigenvector) that defined a one-dimensional vector subspace (i.e., a certain direction in the eightdimensional vector space). These eigenvectors represented a well adapted basis of the eight-dimensional vector space, characterizing the most important directions (in the sense of the VAF), and, in this setting, 
a principal component was simply the projection of the data onto a subspace spanned by a certain eigenvector. The ratio between the first eigenvalue and the sum of all eigenvalues could be viewed as an index of the whole-body coordination (this value is commonly called the VAF by the PC1 and is referred to as PC1\%). A PC1\% value equal to $100 \%$ meant that the trajectory in the space of angles was a straight line (i.e., all angles were linearly correlated together). However, a low PC1\% value indicated that only one principal component could not describe precisely the whole-body movement (i.e., the observations lay in a subspace whose dimension was $>2$, and thus, nonlinear relationships between angles existed). Therefore, we also reported the second PC whose VAF is denoted by PC2\%. Large PC2\% values expressed that at least one angular displacement differed from the most common waveform given by PC1.

Here, instead of using 1560 independent PCAs (a PCA for each trial, each condition, and each participant), we used a PCA whose input dataset consisted of 800 or 960 columns ( 8 angles $\times 10$ trials $\times 10$ or 12 participants) and 200 rows (normalized time) for each experimental condition. The analysis was exactly the same as the one depicted above, except that the number of column of the input dataset changed. In this manner, the PCA automatically extracted the commonality between the shapes of the angular displacements [as in the study by Thomas et al. (2005)]. Nevertheless, individual PCA were also performed when necessary to seek intertrials adaptation, especially during the experiments with equilibrium or spatial constraints.

Reconstruction from PCs. A useful feature of PCA is the possibility to reconstruct an approximated whole-body movement from the data projected on the vector subspace spanned by the first eigenvector (or by more eigenvectors), using the inverse (or equivalently the transpose in our case) of the matrix $W$. Here, the reconstructed centered and normalized angular displacements were simply obtained by the following formula: $\tilde{\Theta}^{\text {Rec }}=\Pi W^{-1}$. For instance, the reconstruction from PC1 only was obtained by using the first column of $\Pi$ and the first row of $W^{-1}$ of the above formula. Then, the reconstructed angular displacements $\Theta^{\text {Rec }}$ were given, for each column $i$, by the following equation: $\Theta_{i}^{\mathrm{Rec}}=s_{i} \tilde{\Theta}_{i}^{\mathrm{Rec}}+m_{i}$. The same procedure was used to reconstruct movements from both PC1 and PC2 (by taking the appropriate rows and columns of $\Pi$ and $W^{-1}$, respectively). Notice that the reconstructed angular displacements $\Theta^{\text {Rec }}$ were exactly equal to $\Theta$ when all PCs were used. Otherwise, we just got an approximation of the original angular displacements.

Once the reconstructed angular waveforms were obtained, the position of markers in plane $X Y$ were then recalculated. To reconstruct the markers kinematics, we assumed that the foot was fixed (which was approximately the case in practice). Head movement was reconstructed assuming that the trajectory of the auditory meatus marker was the one recorded in practice. Notice that this approximation had no influence on the finger trajectory and little influence on the CoM position.

Finally, the same analyses were performed using the covariance matrix rather than the correlation matrix. In particular, this did not change the main results presented below. Here, we considered that the correlation matrix was better suited because angular displacements had significant different amplitudes.

Correlation analyses. Covariation between angles was also estimated by means of a pairwise correlation analysis. Although loadings gave information concerning the joint coupling between pairs of angles (indeed, if loadings were the same for two angles, one could expect that these angles were highly correlated), a more direct method to analyze the coupling between pairs of angles was to compute the correlation coefficients between each pair of angles. In the two-dimensional case (i.e., if we observe only two angles), PC1\% value was correlated to the coefficient of correlation. Thus, this analysis could be viewed as a local analysis compared with the global joint coupling given by the PC1\% value. For a total of eight angles, 28 correlations were computed on all pairs of angles, which corresponded to the correlation matrix coefficients analysis, for each single movement. Statistically significant correlation coefficients $(p<$ 0.05 ) were only retained by means of Matlab corrcoef function.

Numerical simulation. To test the feasibility of a strategy combining an angular covariation with the production of a desired finger trajectory in the Cartesian space ( $\mathrm{S}$ and $\mathrm{C}$ conditions), we conducted a numerical simulation. To this aim, we hypothesized that the movement was optimal according to the minimum angle jerk criterion in the space of elevation angles (Wada et al., 2001). Under this assumption, the angles covariation was perfect (i.e., the VAF by PC1 was exactly equal to $100 \%$ ). This meant that all body angles were driven by one single principal component or, in other words, that one single module ensured both spatial and equilibrium subtasks. This was a plausible assumption according to previous results (Alexandrov et al., 1998; Thomas et al., 2005), which indicated that a single PC captured similar whole-body reaching tasks. In such a task, there was an infinity of final postures allowing to put the fingertip on the target and to preserve equilibrium because there were 8 degrees of freedom. In this simulation, the initial posture was fixed and we used a numerical optimization procedure aimed at finding the final posture such that the resulting finger trajectory fitted at best to the imposed finger path in task space (either a straight line or a circular arc, as in the S and C conditions). This was possible because, once a final posture was selected, the minimum angle jerk criterion allowed us to determine all limb displacements and, therefore, the finger path. The detailed procedure is given in Appendix.

Statistical analysis. All variables were normally distributed (ShapiroWilk's $W$ test) and their variance was equivalent (Levene's test). Statistical effects were tested by performing ANOVAs (repeated measures) when appropriate [either a $5(\mathrm{~B}, \mathrm{~K}, \mathrm{R}, \mathrm{S}, \mathrm{C}) \times 2(\mathrm{D} 1, \mathrm{D} 2)$, a $3(\mathrm{~B}, \mathrm{~K}, \mathrm{R}) \times$ $2(\mathrm{D} 1, \mathrm{D} 2)$, or a $3(\mathrm{~B}, \mathrm{~S}, \mathrm{C}) \times 2(\mathrm{D} 1, \mathrm{D} 2)$ analysis]. Post hoc analyses were conducted with Scheffé's test and Student's $t$ tests were also used.

\section{Results}

\section{Experimental task achievement}

\section{Endpoint accuracy}

Endpoint accuracy of the fingertip was first measured by means of $95 \%$ confidence ellipse analysis, in the sagittal plane. The area of the ellipses was equal to $6 \pm 2.5 \mathrm{~cm}^{2}$ on average, and their major and minor axes had similar lengths. The inspection of the areas of all individual ellipses across all experimental conditions did not reveal significant differences (area of ellipses; ANOVA, $p=0.82$ ). Moreover, the mean endpoint error was $3 \pm 2 \mathrm{~cm}$ in three dimensions, and the LD between the initial finger position and the target were consistent among the different experimental conditions $(p=0.61)$ (Table 1$)$.

\section{Angular variability}

We verified, for each participant and each angle, the consistency of angular displacements, in each experimental condition. The maximum intrasubject SD of amplitudes was $<4^{\circ}$.

\section{Movement duration}

We found similar movement durations for all the experimental conditions (ANOVA, $p>0.2$ for all conditions), except for the semicircular finger path condition in which the movement duration was significantly longer ( $40 \%$; ANOVA, $p<0.05)$ compared with the other experimental conditions (Table 1). This result is logical because the finger path imposed in this condition was obviously longer. However, the average velocities recorded in this condition were similar to those of all the other conditions ( $p=$ 0.23) (Table 1).

\section{Specific experimental constraints}

All participants succeeded in performing the constrained motor tasks (extended-knee, K; reduced base of support, R; straight trajectory, S; and semicircular trajectory, C).

Concerning the equilibrium constraints, as expected, the average knee joint amplitudes recorded in the extended knee condition $\left(5 \pm 2^{\circ}\right)$ were significantly lower $(t$ tests, $p<0.001$, for both distances) than those recorded during the basic motor task (B condition, $50 \pm 20^{\circ}$ ). Furthermore, the CoM position remained significantly higher $(+0.1 \mathrm{~m})$, compared with the basic 
motor task, when participants were requested to keep their knees extended, as a consequence of the lack of knee flexion. The extended knee condition was characterized by an important forward trunk bending (Fig. 3, green histograms for Pe and $\mathrm{Tr}$ ). Moreover, all participants significantly decreased the A-P CoM displacements in the reduced base of support condition ( $t$ tests, $p<0.01$, for both distances). The average decrease was $25 \%$ for the short distance and 50\% for the long distance (Table 2, Fig. 4).

Concerning the spatial constraints, the index of finger path curvature increased significantly ( $t$ tests, $p<0.001$ ) by $\sim 400 \%$ in the imposed semicircular trajectory condition compared with the basic one and remained concave in the sagittal plane (Table 1, Fig. 4). Analysis of index of finger path curvature in the imposed straight finger path condition confirmed that all participants produced approximately straight paths. Path curvature significantly decreased by $75 \%$ compared with those measured in the unconstrained condition $(t$ tests, $p<0.001)$.

Finally, no statistical difference between the two groups of participants that performed the basic motor task was found.

\section{Effect of equilibrium constraints}

Figure 2, $a, b$, and $b^{\prime}$, qualitatively depicts the initial and final whole-body configurations, as well as the CoM and finger trajectories, in the basic (B) and equilibrium constraints conditions ( $\mathrm{K}$ and $\mathrm{R}$ ) for both target distances.

Finger paths in the sagittal plane were strictly concave and approximately similar between the different experimental conditions (ANOVA, $\mathrm{B} \times \mathrm{K} \times \mathrm{R} \times 2$ distances; $p=0.52$ ) (Table 1, Fig. 4 ), although a slight decrease of the index of path curvature was noticed in the reduced base of support condition. Tangential finger velocity profiles were bell-shaped in these conditions, as depicted in Figure 4 . The velocity profiles recorded in the basic motor task were strongly asymmetric: the acceleration duration was shorter than the deceleration duration [average relative time to peak of velocity (i.e., TPV value) was equal to $0.45 \pm 0.05$ in the basic condition] (Table 1). A significant difference was found between the basic and equilibrium conditions (ANOVA, $p<$ 0.05 ), and a post hoc analysis showed that TPV values significantly tended to 0.5 in the extended knee condition $(p<0.05)$, whereas they remained invariant in the reduced base of support condition $(p=0.57)$.

Forward CoM displacements in the basic and extended knee conditions were $\sim 40$ and $70 \%$ of the foot length for the short and long distances, respectively. In contrast, as already noticed, the participants limited the forward A-P CoM displacement $[<4 \mathrm{~cm}$ (i.e., $<30 \%$ of the foot length)] in the reduced base of support condition ( $\mathrm{R})$, because of the experimental restriction; significant statistical differences $(p<0.01)$ were obtained when comparing the reduced base of support condition with the basic and extended knee conditions. Despite these modifications in ampli- tude, CoM path was task independent and kept the same characteristic two components: an initial curvilinear and goal-oriented forward displacement followed by a straight downward movement (Stapley et al., 1999; Pozzo et al., 2002). Downward displacements of CoM were larger in the basic and reduced base of support conditions compared with the knee extended condition $(p<0.01)$ (Table 2). Notice that forward and downward displacements of the CoM significantly increased for the long distance $(p<0.01)$ as a consequence of a greater contribution of shank, pelvis, and humerus segments, necessary to reach the target (Fig. 3, histograms). The velocity profiles of the CoM were single-peaked in all conditions (Fig. 4) and exhibited the same trend as finger TPV values.

Interestingly, participants were able to keep their equilibrium in both conditions. The changes in angular displacements were not detrimental to the covariation of elevation angles. Indeed, PCA showed that the VAF by PC1 was constantly $>85 \%$ for all the conditions ( $p=0.45$ ) (Fig. $5 a$, black histograms), which is comparable with the values calculated in previous similar motor tasks (Alexandrov et al., 1998; Thomas et al., 2005). The PC1\% value increased significantly $(+3 \%)$ at the long distance compared with the short distance $(p<0.01)$. Moreover, the principal component waveforms did not change significantly as reflected by the large correlation coefficients computed when comparing PC1 and PC2 between conditions (Fig. 5b). The PC1 captured the monotonic angular displacements with bell-shaped velocity pro- 
D1

Trajectory

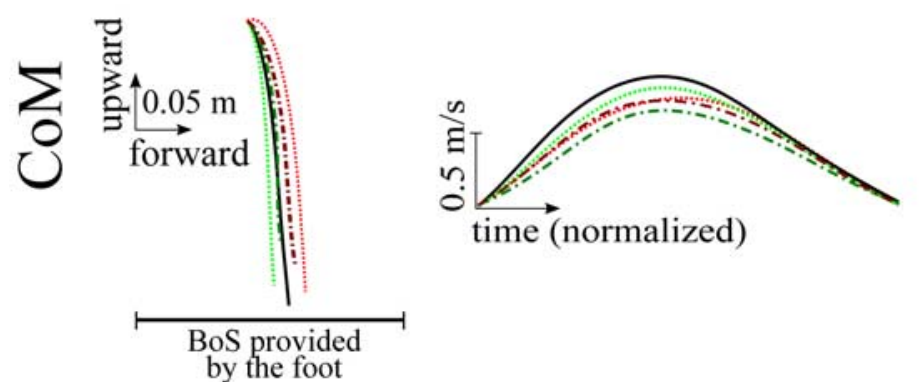

by the foot
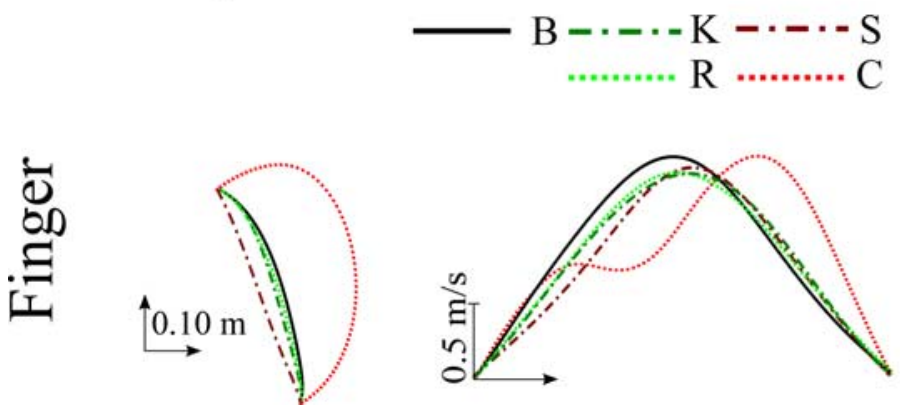

D2

Trajectory

Velocity
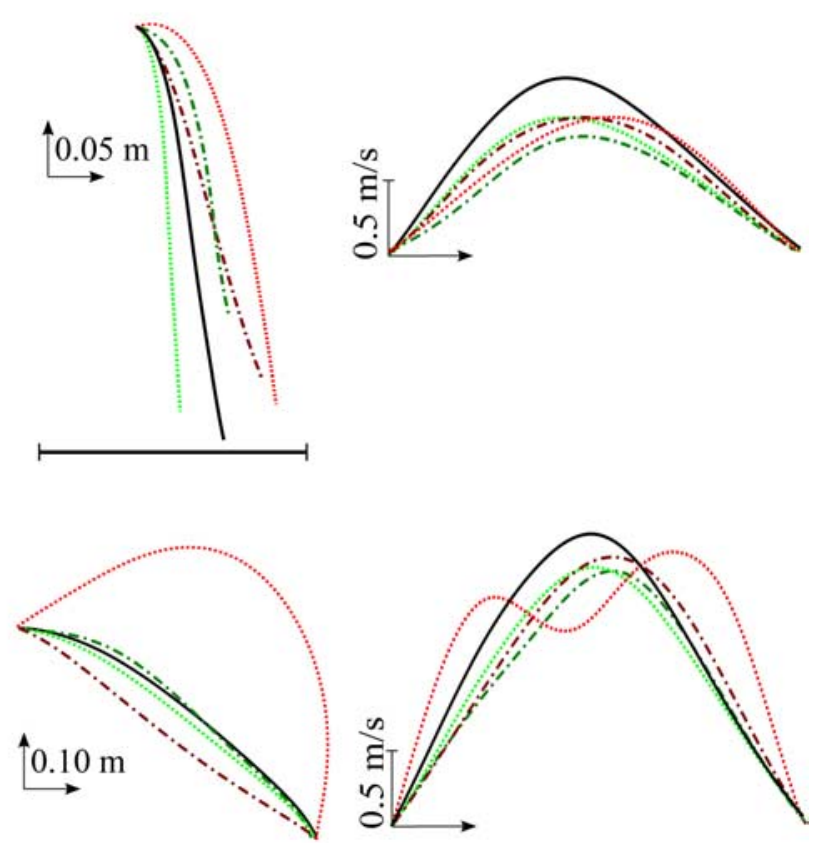

Figure 4. Average CoM and finger trajectories and tangential velocity profiles, in the sagittal plane, for all experimental conditions: basic (B), extended knee (K), reduced base of support (R), straight finger trajectory (S), and semicircular finger trajectory (C). SDs are not depicted for the sake of clarity.

files, whereas the second PC captured nonmonotonic angular displacements with double-peaked speed profiles (Fig. $3 a, b$, middle column). In addition, an inspection of the loadings did not reveal significant changes in the reduced base of support condition for both distances (average $r^{2}=0.97$ ). At variance, in the extended knee condition, the angles were differently distributed among PC1 and PC2, and showed changes mainly in the leg angles (e.g., shank and thigh), because of the joint restriction imposed at the knee (Fig. $5 c$ ). However, the $\mathrm{PC} 1 \%$ value remained high despite these changes.

\section{Effect of spatial constraints}

Figure 2, $c$ and $c^{\prime}$, qualitatively illustrates the initial and final whole-body configurations, as well as the CoM and finger trajectories, under spatial constraints ( $\mathrm{S}$ and $\mathrm{C}$ ) for both target distances.

As already noticed, participants performed approximately straight and semicircular finger paths in these conditions. The velocity time courses tended to become more symmetric in the imposed straight finger trajectory condition (TPV value was on average $0.49 \pm 0.04 ; p=0.06$ ), being close to what would be predicted by a minimum Cartesian jerk model (straight trajectory and symmetric speed profile) (Flash and Hogan, 1985). In contrast, the velocity profiles recorded in the semicircular condition were double-peaked (Table 1, Fig. 4) similar to those obtained during via point movement (Viviani and Flash, 1995).

The analysis of CoM displacements revealed significant changes across the experimental conditions (Table 2, Fig. 4). Specifically, in the imposed finger trajectory conditions, A-P and vertical CoM displacements increased $(+22 \% ; p<0.05)$ and decreased $(-12 \% ; p<0.01)$, respectively, compared with the basic condition. These changes led the participants to adopt a more risky inverted pendulum configuration because of (1) the A-P CoM displacement close to the forward limit of the BoS and (2) a longer level arm inducing a larger potential energy and a greater whole-body momentum (approximately $+22 \%$ ) (Table 2) compared with the basic experiment. In contrast to finger trajectory, the CoM velocity remained single-peaked in $\mathrm{C}$ condition.

Remarkably, the covariation of elevation angles was strongly affected by spatial constraints onto the finger path. The statistical analysis revealed a significant decrease of the $\mathrm{PC} 1 \%$ value in these conditions $(-8$ and $-13 \%$, respectively; $p<0.01)$ compared with the basic condition. This was compensated by a drastic increase of the $\mathrm{PC} 2 \%$ value that was about twice larger than the values computed in the basic condition (Fig. 5, gray histograms). Similarly to the basic motor task, the covariation of elevation angles was greater $(+6 \%)$ for the long distance compared with the short distance $(p<0.05)$ in the motor tasks with spatial constraints. The inspection of principal components revealed that their waveforms were unchanged compared with the basic condition (Fig. 5b, large correlation coefficients). Nevertheless, the loadings analysis revealed changes in their distribution among the PC1 and especially the PC2. A noticeable effect was an increase (in absolute value) of the loadings for the arm angles (humerus, forearm, and hand): the upper limb angles were more strongly captured by the PC2 in the imposed finger trajectory conditions compared with the basic one. This result was quite striking because these changes were accompanied by a significant decrease and increase of the PC1\% and PC2\% values, respectively. Thus, spatial but not equilibrium constraints modified significantly the relative importance of PC1 and PC2.

In particular, an interesting result was the decrease of the 
PC1\% and the increase of PC2\% values (14 of 14 participants for the short distance; 12 of 14 participants for the long distance) consecutively to the straight finger path constraint. To evaluate a possible effect of a learning process on this change in the covariation of elevation angles, we asked 4 among the 14 participants to perform 50 successive trials in the straight Cartesian trajectory condition for the long distance. To avoid the potential influence of fatigue, participants took a rest of $1 \mathrm{~min}$ after each block of 10 trials (Schmid et al., 2006). Figure 6 shows average results for the PC1\% value. A linear regression analysis on the average PC1\% values among the 50 repetitions showed slope and $r^{2}$ coefficients $<10^{-3}$ indicating a lack of adaptation among the 50 trials in the imposed straight finger trajectory condition, for the long distance (SD2). Furthermore, a between-participants $t$ test comparison among the PC1\% average values of the 10 first and 10 last trials did not give significant differences $(p=0.29)$.

\section{Motion reconstruction from PCs}

To better elucidate the respective roles of the PC1 and PC2 on the movement, we reconstructed the angular displacements and then the kinematic features of the body markers, from either the first or both the first and the second principal components (see Materials and Methods).

First, the whole movement was reconstructed based on the data contained in the first principal component. Figure 7 shows that the reconstructed movement was very similar to the one recorded in the basic condition (B), for a typical participant. The characteristic features of the basic motor task were captured well by the PC1 (e.g., the forward CoM projection and the concave finger trajectory) (Table 3) (Pozzo et al., 2002). In particular, the CoM and finger trajectories were close to the recorded ones (Fig. 7a), the angular displacements were also similar as indicated by the large correlation coefficients (Fig. $7 b$ ), and the velocity profiles of both the finger and the CoM were single-peaked (Fig. 7c). Second, when the motion was reconstructed using the data contained in the PC1 and PC2, the whole-body movements became extremely close to the recorded ones, whatever the experimental conditions (as proved by the high correlation coefficients reported in Fig. $7 b, c)$.

However, the main result lies in the differences observed when reconstructing motions from $\mathrm{PC} 1$ in the motor tasks with spatial constraints imposed on finger trajectory. Movements reconstructed in these conditions showed that the requested Cartesian constraints were not captured by the PC1 (i.e., the straight and

$b$ loadings of a constrained condition.
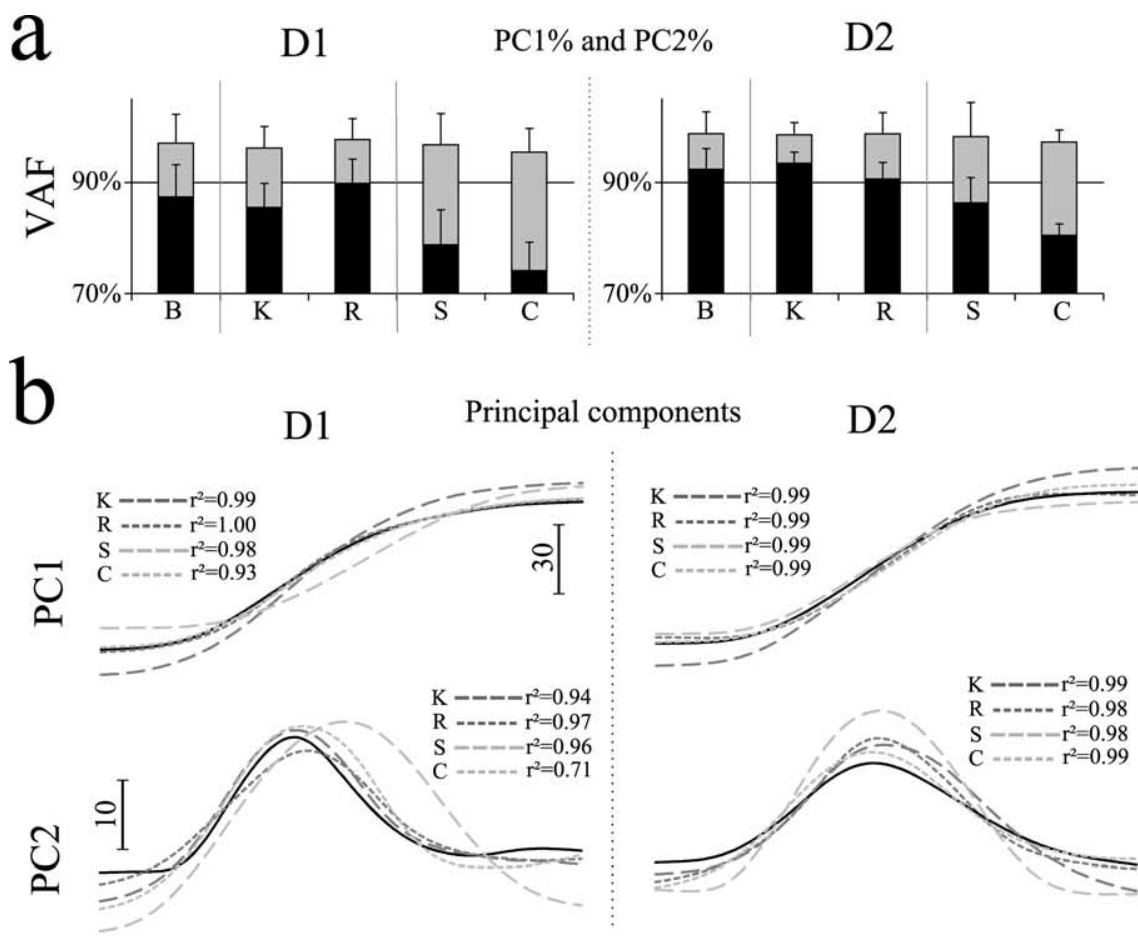

C

D1

B -

K

$\mathrm{R}$

S

C

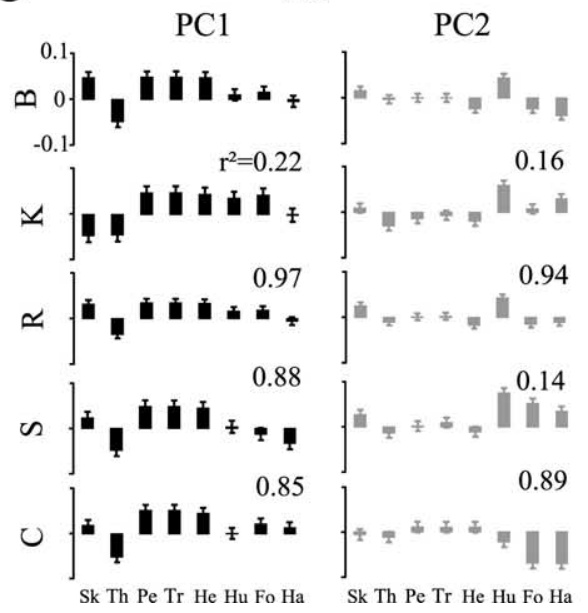

\section{Loadings}

D2

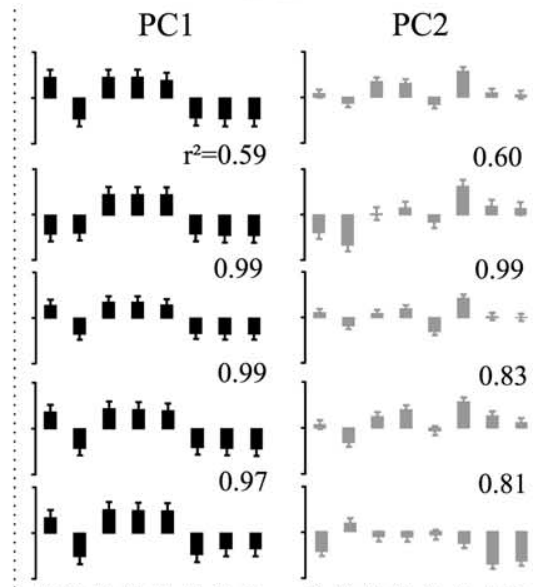

Sk Th Pe Tr He Hu Fo Ha Sk Th Pe Tr He Hu Fo Ha

Figure 5. $\boldsymbol{a}, \mathrm{PC} 1 \%$ and $\mathrm{PC} 2 \%$ values. Means and SDs of the percentage of variance explained by the first two principal components computed for the elevation angles, for the two target distances (D1, left histograms; D2, right histograms) and for each experimental condition. The black and gray bars represent the VAF by the first $\mathrm{PC}$ and the VAF by the second $\mathrm{PC}$ (cumulative histogram), respectively. $\boldsymbol{b}$, Principal components. The $\mathrm{PC} 1$ is the most common angular waveform found in the dataset and PC2 is the second angular waveform. Each principal component obtained in the basic $(B)$, equilibrium $(K, R)$, and spatial $(S, C)$ constraints motor task is depicted. c, Loadings. Mean values, for both PC1 (black) and PC2 (gray), and for all experimental conditions. Error bars show the SD. Correlation coefficients $\left(r^{2}\right)$ are reported between the loadings of the basic condition and the

semicircular finger paths constraints were not encoded in the PC1) (Fig. $7 a$, second and third columns). Table 3 shows, on specific parameters, that the A-P CoM displacement was well reconstructed by the use of $\mathrm{PC} 1$ in all experimental conditions, in contrast to the finger trajectory. Indeed, the index of finger path curvature did not fit well with those recorded experimentally (or, equivalently, obtained when reconstructing the motion from all PCs). Whatever the condition, the reconstructed finger path was 


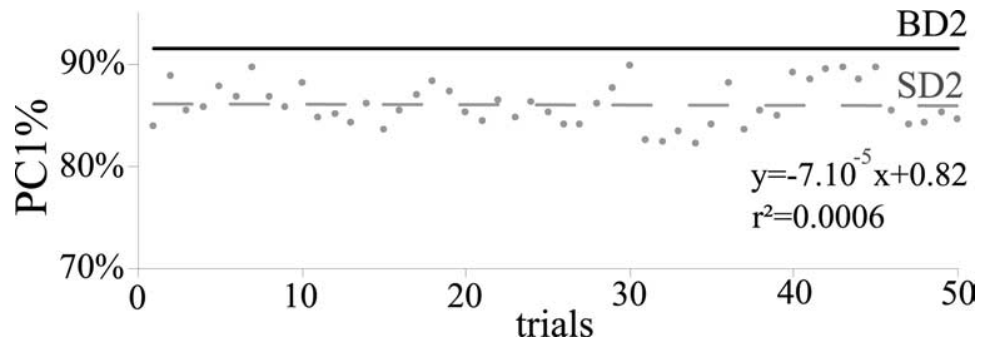

Figure 6. Typical PC1\% values of one typical subject for 50 successive trials. The continuous black line corresponds to the PC $1 \%$ baseline measured during the basic condition. The dashed line indicates the linear regression calculated on the 50 records performed in the straight finger trajectory condition at long distance (SD2).

constantly slightly concave and, in particular, did not fit with the spatial constraints. The addition of PC2 in the reconstruction process was necessary to recover the spatial requirements imposed to the motor task (Table 3 ). Therefore, the PC2 appeared to be strongly implied in a fine control of the fingertip path, whereas the PC1 encoded the main characteristics of the whole-body motion performed in the basic condition, including the forward CoM displacement and the slightly concave finger path. Correlation analyses between the recorded and reconstructed angular displacements were also performed to quantify in which proportion each angular displacement was reconstructed. Lower correlation coefficients were measured between angular displacements for the angles of upper limbs, especially in the semicircular condition. This result suggests that PC1 primarily described the equilibrium and free reaching subtasks, whereas PC2 (for which PC2\% values increased significantly in the spatial constraints conditions) adjusted the finger trajectory to the task requirement ( $\mathrm{S}$ and $\mathrm{C}$ conditions). Such a reorganization was mainly achieved by means of the hand, forearm, and humerus angular displacements. These findings were consistent for both distances and across participants, even for those who maintained a relatively high joint coupling during the straight finger trajectory condition.

\section{Local coupling}

To uncover specific angles covariations at different levels of the whole body, we performed a correlation analysis between each pair of angles (see Materials and Methods) (Fig. 8a). Because eight angles were measured, a total of 28 correlation coefficients were analyzed. In most cases, the results showed that all correlation coefficients were $>0.7$ in the basic and equilibrium constraint motor tasks (see top left polar graph), as expected from the results given by PCA in these conditions (Fig. 5). An ANOVA performed on the 28 coefficients ( 28 factors), for each condition separately, showed a significant main effect $(p<0.001)$ (i.e., at least two correlation coefficients were significantly different among the 28 comparisons). Despite the fact that all correlation coefficients were $>0.7$, it was noticeable that, when calculating correlations between angles of the upper limbs (U) with angles of the lower limbs (L), the correlation coefficients were slightly lower (Fig. 8, mixed pairs of angles, L/U, M1 to M15) than the coefficients corresponding to L1 to L10 (L/L, pairs of angles within the lower limbs group). Nevertheless, a post hoc analysis did not reveal important differences (in general, $p>0.05$ ) for the basic and equilibrium constraints conditions.

At variance, in the spatial constraints conditions (Fig. $8 a$, top right polar graph), the correlation coefficients significantly decreased for the mixed pairs of angles (L/U, M1 to M15, <0.7).
Post hoc analysis proved that all coefficients of this group (L/U, M1 to M15) were significantly different from both the upper limbs (U/U, U1 to U3) and the lower limbs (L/L, L1 to L10) comparisons $(p<0.05$ at D1), illustrating a change in the wholebody motor strategy (a significant separation between upper and lower limbs). For the long distance (Fig. $8 a$, bottom right part), correlation coefficient values increased compared with the short distance condition $(p<0.01)$, indicating a stronger coupling of upper and lower limbs when the task required a fine equilibrium control (long distance, D2). It should be noted that the decrease of the covariation of elevation angles, observed in the straight (S) and semicircular (C), was less pronounced at D2 compared with the short distance (D1), remaining, however, below the values obtained in the basic and equilibrium constraints motor tasks (Fig. $8 a$, left).

To verify the idea of a dissociation of reaching limbs (arm segments) from a robust equilibrium module during spatial constraint, we computed the average correlation coefficients between the waveforms measured in the basic motor task (B) and the other experimental conditions, for each of the eight angles (Fig. $8 b$ ). In this way, we quantified the changes in the angular displacements induced by the experimental conditions with respect to the basic condition. A repeated-measure ANOVA showed that these changes were significantly concentrated in the hand, forearm, and humerus angles compared with the leg, trunk, and head angles $\left(p<0.001\right.$ and values of $r^{2}=0.92$ and 0.72 for lower limbs and upper limbs, respectively). Thus, in addition to the drastic reorganization of upper limbs in response to the spatial constraints, the lower limbs displacements remained invariant (i.e., highly correlated to the ones observed in the basic condition).

Overall, these analyses revealed one PC that captured lower limbs and CoM displacements and resisted to spatial constraints exerted on the finger, and another one, task-dependent, that emerged when focal subtask became a priority.

\section{Simulation}

The previous results showed that, in the basic condition, equilibrium and focal subtasks were integrated in the same module from which emerged an additional module when specific finger path had to be planned. Because priority was given to finger trajectory without requirement concerning the joint strategy to be used (i.e., one could have asked the participants to produce a straight finger path and to move all body segments at the same time), the dissociation of equilibrium and focal task goals in two modules could be inherent to the spatial constraints. Thus, to test the plausibility of one single module to generate simultaneously a straight path in angle space and a specific finger trajectory in Cartesian space, we performed a simulation (for details, see Materials and Methods and Appendix).

Therefore, we simulated the integration of both the equilibrium and reaching task objectives in one single module during whole-body pointing (WBP) under spatial restriction. The result of this simulation showed that the production of a perfectly straight trajectory in the elevation angles space, combined with an almost straight or semicircular finger path in Cartesian space is (1) possible with respect to the physiological ranges of joint angular displacement but (2) detrimental to equilibrium control. In particular, the spatial constraints led to a CoM displacement close 
a

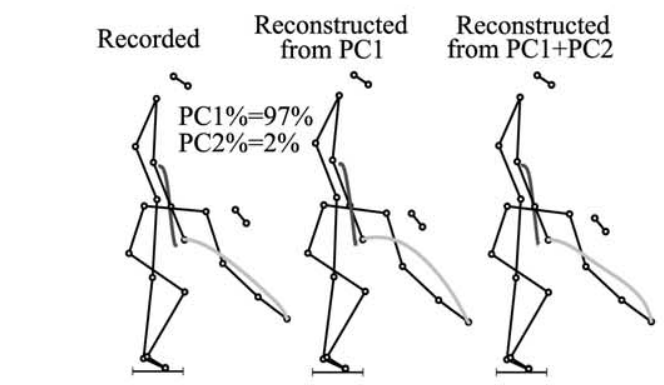

$\mathrm{b}$
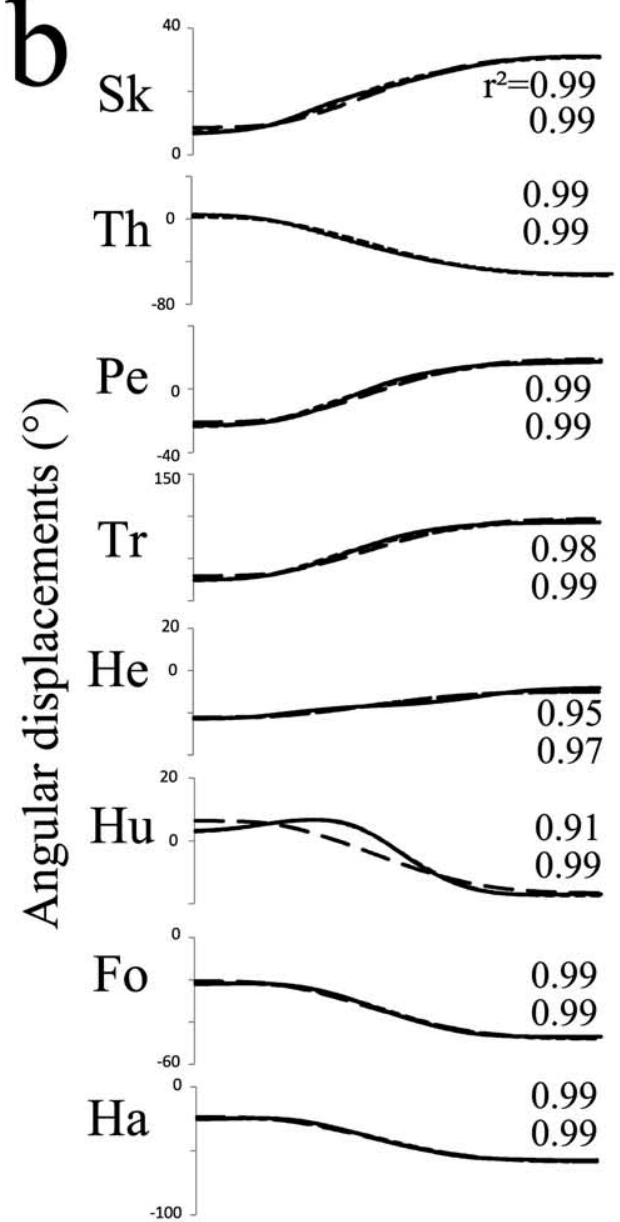

$C$

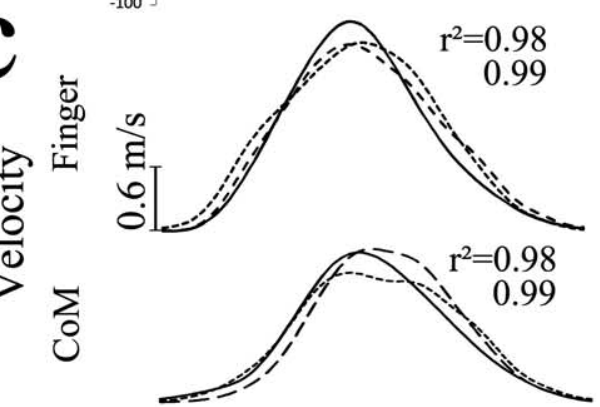

time (normalized)

Recorded
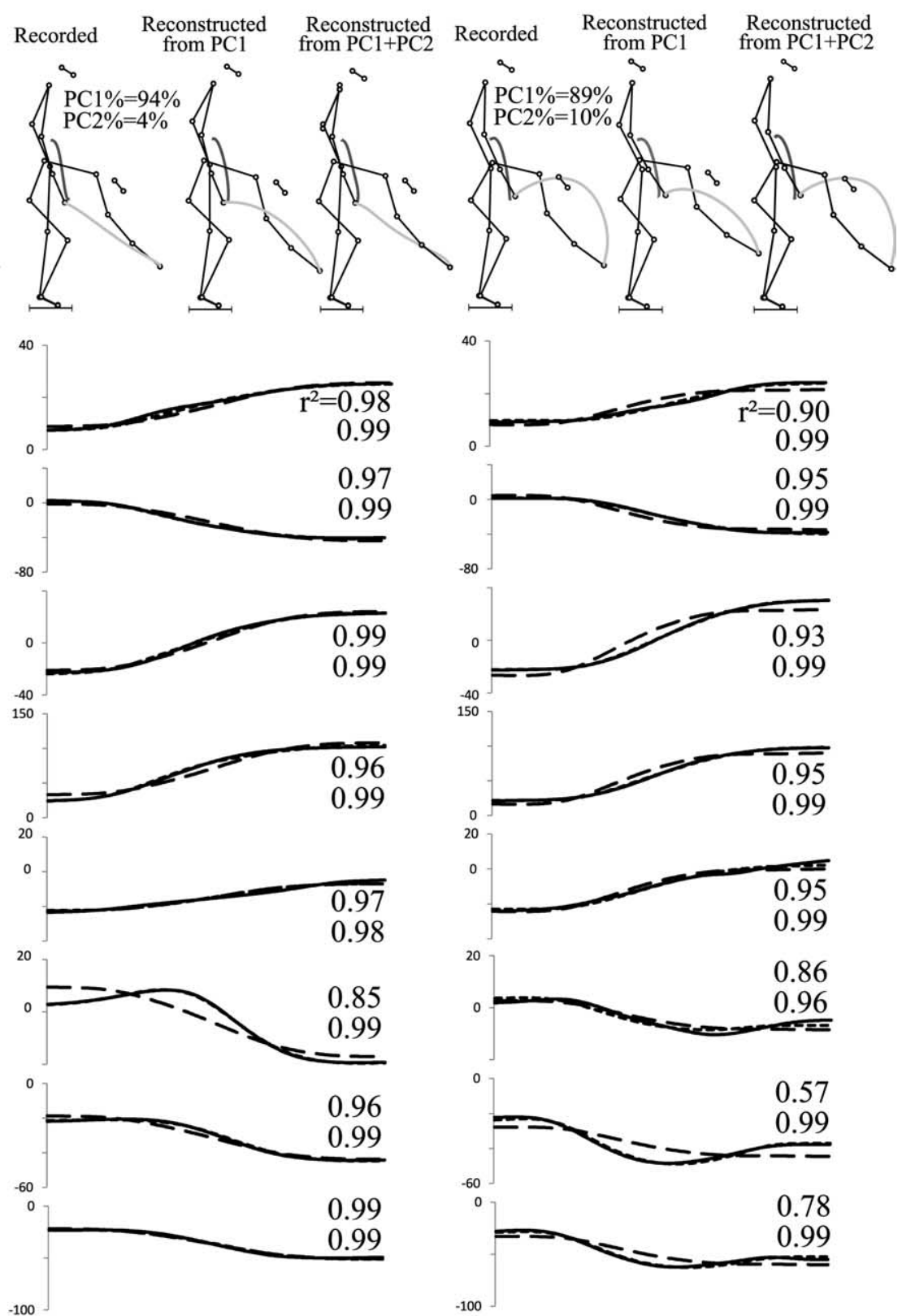

$-100$
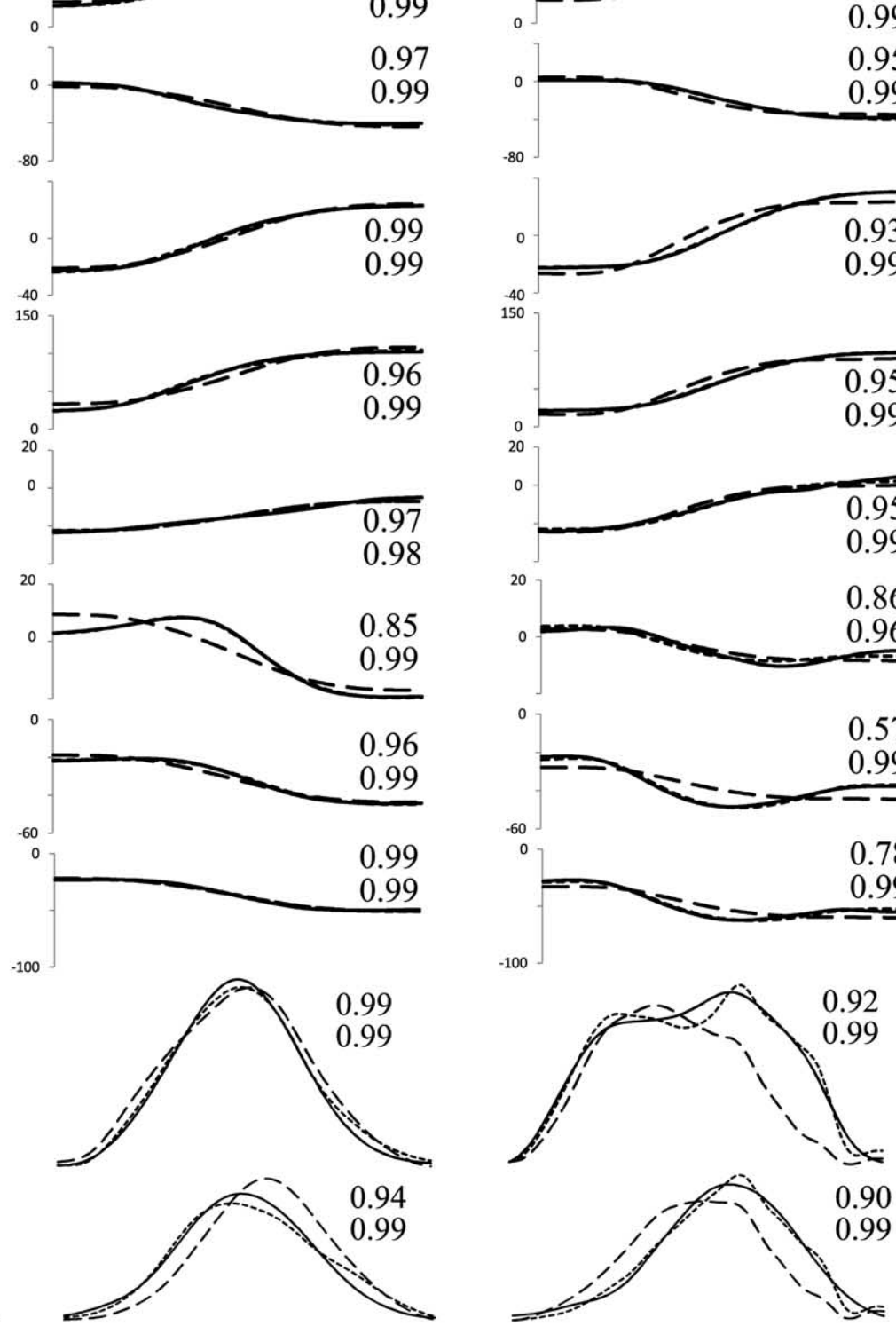

$-100$

\section{Semi-circular}

- Reconstruction from PC1+PC2

Figure 7. a, From left to right, stick diagrams depicting the finger and $\mathrm{CoM}$ trajectories of a recorded and typical movement, the corresponding reconstruction from $\mathrm{PC} 1$, and from both $P C 1$ and $P(2$ for the following conditions: basic task (B), imposed straight finger trajectory condition (S), and semicircular finger path condition (C). $\boldsymbol{b}$, Recorded and reconstructed angular displacements from $P C 1$, and both $P C 1$ and $P C 2$, across the spatial constraints condition. For the reconstruction from $P C 1$ and $P C 2$, the displacements are almost superimposed and not always visible. Correlation coefficients $\left(r^{2}\right)$ are reported: the first and second values are the results of comparisons between the basic angular displacement and the reconstructed angular displacement from $\mathrm{PC} 1$ and from both $\mathrm{PC} 1$ and $\mathrm{PC}$, respectively. $c$, Comparison between recorded velocity profiles and reconstructed velocity profiles from either PC1 or both PC1 and $P C 2$. Correlation coefficients are reported, measuring the similarity between these velocity profiles. The first and second values correspond to a comparison with the reconstructed movement from $\mathrm{PC} 1$ and from both $\mathrm{PC} 1$ and $\mathrm{PC}$, respectively. 
Table 3. Reconstructed kinematic measures from all PCs, from PC1 only, and from both PC1 and PC2

\begin{tabular}{|c|c|c|c|c|c|c|c|c|c|}
\hline & \multicolumn{3}{|c|}{ A-P CoM displacement (m) } & \multicolumn{3}{|l|}{ IPC } & \multicolumn{3}{|l|}{ VAF } \\
\hline & All PCs & PC1 only & $P C 1$ and $P C 2$ & All PCs & PC1 only & $P C 1$ and $P C 2$ & All PCs & PC1 only & $P C 1$ and $P C 2$ \\
\hline B & $0.08 \pm 0.02$ & $0.08 \pm 0.02$ & $0.08 \pm 0.02$ & $0.11 \pm 0.05$ & $0.17 \pm 0.06$ & $0.13 \pm 0.06$ & 1.0 & $0.91 \pm 0.05$ & $0.99 \pm 0.01$ \\
\hline$S$ & $0.10 \pm 0.02$ & $0.09 \pm 0.02$ & $0.10 \pm 0.02$ & $0.03 \pm 0.01$ & $0.16 \pm 0.05$ & $0.06 \pm 0.04$ & 1.0 & $0.86 \pm 0.06$ & $0.98 \pm 0.02$ \\
\hline C & $0.11 \pm 0.03$ & $0.12 \pm 0.05$ & $0.11 \pm 0.04$ & $0.49 \pm 0.21$ & $0.24 \pm 0.19$ & $0.49 \pm 0.20$ & 1.0 & $0.80 \pm 0.03$ & $0.97 \pm 0.03$ \\
\hline
\end{tabular}

Average values (and SD) across participants of anteroposterior $\mathrm{CoM}$ displacement $(\mathrm{A}-\mathrm{P} \mathrm{COM})$, index of finger path curvature $(\mathrm{IPC})$, and VAF by all $\mathrm{PC}$, the first $\mathrm{PC}$ and both $\mathrm{PC} 1$ and $\mathrm{PC} 2$, in the spatial constraints conditions ( $\mathrm{S}$ and $\mathrm{C})$, at the long distance (D2).

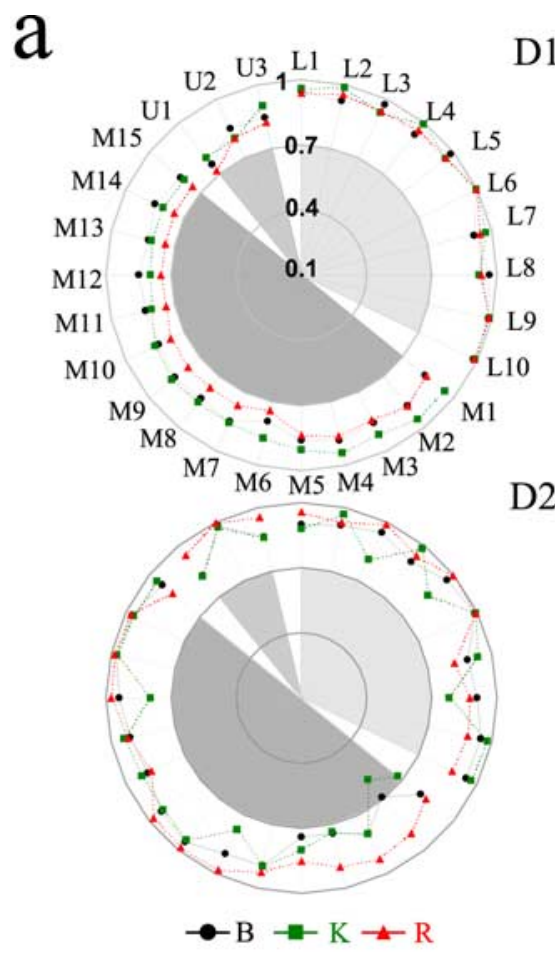

D1

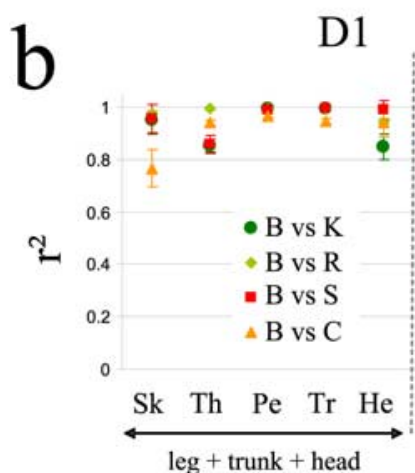

D1

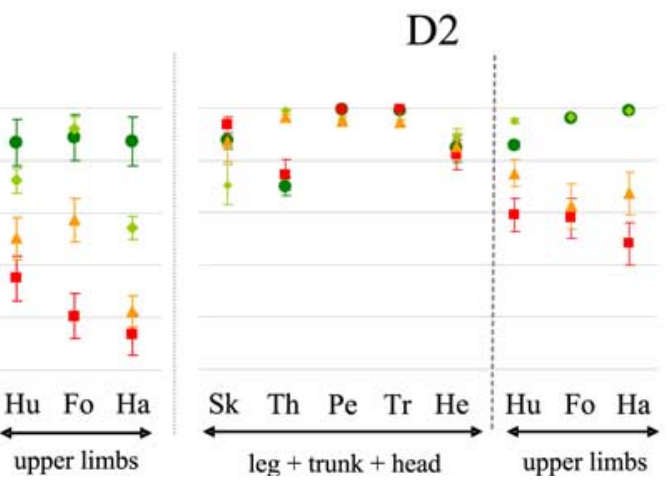

Figure 8. a, Polar diagrams of average correlation coefficients computed for the 28 pairs of elevation angles. The center and the external border of each polar diagram correspond to a minimal $(=0.1)$ and maximal correlation $(=1)$, respectively. SDs are not displayed on the figure for the sake of clarity. Values for the two target distances (short distance, D1, top; long distance, D2, bottom) and for the two main experimental constraints (left, Kand R for the two equilibrium constraints; right, S and ( for the two finger trajectory constraints). The legend on the right indicates the angles defining each pair considered. $\boldsymbol{b}$, Average correlation coefficients computed between the time series of angular displacement recorded during the basic condition (B) and the other conditions (K, R, S, and C), for each angle, at D1 (left) and D2 (right). Error bars represent the SE between participants. The abbreviations are the same as in Figure 2.

to the boundaries of the base of support (90 and 100\% of the base of support, respectively) (Fig. $9 a-c$ ). Indeed, the backward CoM displacement toward the heels recorded in the straight finger path simulation (Fig. 9b) reduced the balance safety margin. Furthermore, a second forward CoM acceleration toward the target to reach requires a large energy consumption. At last, back-and-forth CoM displacements drastically differed from the robust monotonic CoM forward displacements presently recorded in all experimental conditions.

\section{Discussion}

Our results show that reaching and equilibrium subtasks are integrated in one single coordinative structure (i.e., one single module) when subjects reached beyond arm's length without any particular spatial or equilibrium constraint. In contrast, when the CNS has to explicitly plan the finger path, the basic kinematic strategy was separated in two modules enabling fast adaptation to equilibrium and focal task demands. Here, we discuss the possible origins of arm and lower limbs covariation, and thus we emphasize the decomposition of the single kinematic module in equilibrium and reaching modules.

\section{A single coordinative structure resilient under equilibrium constraints}

Several factors can contribute to build a strong joint covariation. During the present task, the whole body moves downward mainly under the action of gravity. An anticipative inhibition of the tonic activity of the trunk and lower limbs extensors has been described during a similar downward whole-body motion (Cheron et al., 1997), as a general deactivation of extensor muscles [the socalled Hufschmidt phenomenon (Hufschmidt and Hufschmidt, 1954)]. Passive mechanical effects might additionally help in joint coupling. Indeed, previous studies showed that the mechanical consequences of forward arm reaching from an upright stance position consisted of passive forward trunk bending and knee flexion (Ramos and Stark, 1990; Eng et al., 1992; Pozzo et al., 2001), similar to these observed during the present WBP task. Coordinative structures of redundant degrees of freedom can also be accounted for by attractor dynamics, operating on an internal model of the underlying kinematic system (Mussa Ivaldi et al., 1988; Mohan and Morasso, 2006). At last, neural organization as well could determine the present angular covariation. Ivanenko et al. (2005), for instance, identified only one synergy from 


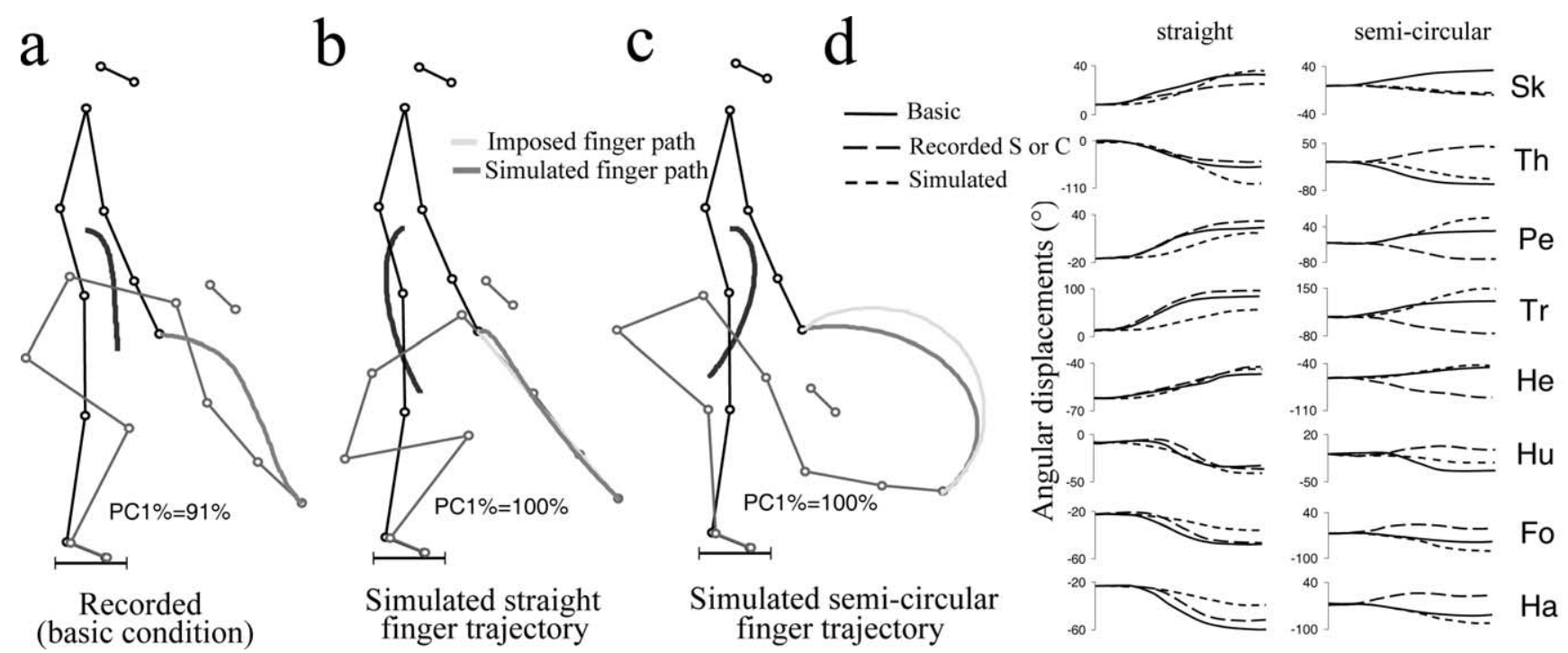

Figure 9. Recorded data. $\boldsymbol{a}$, Typical stick diagram of a whole-body pointing task performed in the basic condition, at long distance (BD2). The black and gray traces depict the CoM and the finger trajectory in the sagittal plane, respectively. Simulated data. $\boldsymbol{b}$, Motor strategy obtained by imposing both a perfect covariation of the elevation angles and a straight finger path. $\boldsymbol{c}$, Motor strategy obtained by imposing both a perfect covariation of the elevation angles and an exaggerated curved finger path. $\boldsymbol{d}$, Angular displacements of elevation angles obtained for the (dark line) recorded data, and simulated data of the straight $(S)$ and semicircular (C) conditions (dotted line). The abbreviations are the same as in Figure 2.

the recorded activity of 32 muscles during a stoop in place task. Moreover, it has been shown that trunk bending (as an important aspect of the present task) was under the control of "axial synergies" (i.e., a coordinated and opposing displacements of trunk and lower body segments) (Babinski, 1899; Alexandrov et al., 1998, 2001b). The present joint coupling is compatible with the functional mechanism previously proposed by which descending neural command related to the desired CoM trajectories and final finger position are transformed into a specific joint angle covariation (Ivanenko et al., 2005; d'Avella et al., 2006; Ting, 2007). For instance, when knee extension was imposed ( $\mathrm{K}$ condition), the pointing was mainly attributable to forward trunk bending to compensate for the lack of knee flexion. This new joint configuration induced a higher level of the position of the CoM as a consequence of the lack of knee flexion, and represented an additional source of postural instability. Surprisingly, these changes did not affect joint coupling compared with the basic condition (B), revealing a significant flexibility of the motor command to keep joint covariation and consecutive similar finger and CoM trajectories in the sagittal plane. Furthermore, when placed on a thin support (R condition), subjects still exhibited strong covariation of elevation angles. Thus, the well preserved joint covariation, the associated stereotypical CoM, and finger trajectories are compatible with a process that would map low-dimensional task variables (the final finger position and the CoM trajectory) onto high-dimensional anatomical details (individual limbs) (Ting, 2007). In the same vein, covariation of limb rotation in extrinsic space points to a simple organization previously identified for the lower limbs (Lacquaniti et al., 1999) or the head segment (Pozzo et al., 1990) during locomotion that both reduces the high dimensionality of the kinematic chain and allows the control of the CoM displacement with respect to gravity. This strategy likely facilitates the integration of reaching and equilibrium subtasks in the same motor plan. Furthermore, because of the mechanical link existing between upper and lower body parts, separate controllers seem not functionally appropriate to predict and compensate the interaction torques inherent to multijoint movements. Pigeon et al. (2003) also demonstrated that, during turn and reach movement involving the trunk, the different body parts are integrated in a single frame of reference to predict appropriate joint torque and prevent disturbing effect on hand path. Finally, the strong temporal coupling between times to peak velocity of the finger and the whole-body center of mass, regardless of drastic change in sensory and mechanical context (earth versus microgravity) previously described for the same task (Patron et al., 2005) (Fig. 7; Tables 1, 2), support the idea that the two task objectives may to some degree share common neural commands.

\section{Decomposition of the coordinative structure in two modules under spatial constraints}

An important result is that, when subjects were forced to move the finger along a straight ( $\mathrm{S}$ condition) or a semicircular $(\mathrm{C}$ condition) path, a second coordinative structure dedicated to arm segment coordination emerged from the initial kinematic strategy. For the exaggerated $\mathrm{C}$ condition, one could have expected such a dissociation because of the large discrepancy between natural and imposed finger trajectories. In contrast, it was more unlikely for the $S$ condition that imposed a straight line close to the basic slightly curved finger path. This result demonstrates that the curved finger path recorded in the free condition was not the result of an error in trajectory planning. Thus, if desired, participants could execute the WBP task by adopting another reaching movement, a straight hand path for instance that is not the one chosen in the free condition.

In $\mathrm{S}$ and $\mathrm{C}$ conditions, priority was given to the control of hand movement, as during object manipulation or object avoidance while reaching performed from a standing posture. Thus, inefficiency in controlling the finger path and the current highly redundant body joint system at the same time rather than a voluntary strategy adopted by the participants, could explain arm and lower limbs decoupling. Several arguments contradict with such a hypothesis. First, the unstable high inverted pendulum posture adopted by the participants when spatial restrictions were present required an efficient equilibrium control. Thus, a precise prediction of the mechanical effect of the CoM acceleration must be incorporated in the 
inverse model of the motor plan to produce a continuous smooth and linear finger path. Because participants succeeded after only two or three trials, without increasing movement duration compared with the free condition or any adaptation after several repetitions (Fig. 6), we conclude that kinematic decoupling was actively performed. Second, simulation procedures have clearly shown that the two subtasks could be integrated in the same motor plan with only few changes in experimentally recorded body geometry, supporting the biomechanical feasibility of keeping both joint covariation and straight finger path. However, CoM analysis revealed that such a strategy was detrimental to equilibrium control, a likely reason for the CNS not to adopt this way. Additionally, this postural solution contrasts with the stereotyped trajectory recorded whatever the condition (Fig. 4) and characterized by an initial forward CoM acceleration. The robustness of this pattern, existing among a wide range of voluntary movements (Crenna and Frigo, 1991; Stapley et al., 1998), could represent a neural constraint contributing to preserve the equilibrium module invariant when reaching under finger path restriction. Consequently, rather than revealing inefficient coordination of the multijoint system because of the experimental condition, we propose that the dissociation of the basic coordinative structure in two modules would correspond to an intended strategy.

Finally, the spatial constraints did not destroy the local coupling of lower segments indicating that postural module was task independent. Instead, a new module used to produce an optional finger trajectory ( $\mathrm{S}$ or $\mathrm{C}$ ) was added up to the module found in the basic whole-body reaching condition as predicted in the top right part of Figure 1.

The present addition of a new kinematic module to an existing one is consistent with the proposal that compound movements are generated by means of a combination of motor programs (Bizzi et al., 2008). Similarly, other studies have demonstrated modularity of muscle use in cat (Ting and Macpherson, 2005; Krouchev et al., 2006), in frogs (Hart and Giszter, 2004; d'Avella and Bizzi, 2005), and in humans (Ivanenko et al., 2005; d'Avella et al., 2006). Basic synergies built during development are probably slowly adjusted and differ slightly their expression to tolerate a wide range of tasks inducing various CoM and endpoint trajectories. However, in an unusual condition, it seems that they can adapt very rapidly or can be completed with additional ones to provide more behavioral flexibility. At which stage of the CNS the motor synergies are adapted to environmental demands or combined remains an open question. Even far from the scope of this study, we would like to discuss briefly some points according to the literature. Capaday (2004) has emphasized the functional linking of motor cortical points based on disinhibition process. Thus, synergies could emerge from the recruitment of various motor cortical points by selected excitation as well as by selected release from inhibition. At the level of the spinal cord, central descending pathways could influence and modulate reflex pathways depending on the context within which we must act (for review, see Dietz, 1992). Various descending pathways can then modulate the spinal cord for greater flexibility of basic reflexes. For instance, recent data recorded in cat showed that neurons in pontomedullary reticular formation can both ensure integrated or independent control of posture and reaching (Schepens and Drew, 2004; Schepens et al., 2008). Together, one can speculate that the basic coordinative structure could first emerge at the level of M1 depending on task demands. To provide flexibility, the motor commands could be adapted at the level of spinal cord via excitation or inhibition processes channeling appropriate contexts to the kinematic strategy and thus constructing the reaching and equilibrium subtasks by using one or two modules. Nevertheless, without a detailed analysis of patterns of focal and postural muscle activity, definite conclusions relating to the organization of descending neural commands are difficult to make. An upcoming study of muscle activation patterns should reveal how many muscle synergies are required for task performance and their robustness to different contexts.

\section{Appendix}

This appendix gives details concerning the kinematic simulation that we briefly described in Materials and Methods.

Let us consider a rigid nine-segment model of the body and define eight elevation angles (Fig. 2, inset) as follows: $\left(\theta_{\mathrm{Sk}}, \theta_{\mathrm{Th}}, \theta_{\mathrm{Pe}}, \theta_{\mathrm{Tr}}, \theta_{\mathrm{He}}, \theta_{\mathrm{Hu}}, \theta_{\mathrm{Fo}}, \theta_{\mathrm{Ha}}\right)=\Theta$.

The foot angle was neglected because ankle marker coordinates remained constant.

First, we assumed that the trajectory in angle space resulted from the minimum angle jerk criterion, which minimized the following integral cost (Wada et al., 2001):

$$
\frac{1}{2} \int_{0}^{\mathrm{MD}} \sum_{k=1}^{k=8}\left(\frac{d^{3} \Theta_{k}}{d t^{3}}\right)^{2} d t
$$

where $\Theta_{k}$ was the $k$ th component of $\Theta$, and $t$ denoted the time.

In the following, the movement duration was normalized $(\mathrm{MD}=1)$. It is well known that the optimal solutions are straight lines in the eight-dimensional vector space connecting the initial configuration of segments $\left(\Theta^{i}\right)$ to the final one $\left(\Theta^{f}\right)$ with the following formula:

$$
\Theta(t)=\Theta^{i}+t^{3}\left(10-15 t+6 t^{2}\right)\left(\Theta^{f}-\Theta^{i}\right) .
$$

Notice that, therefore, the PC1\% value was equal to $100 \%$.

Then, the finger trajectory was given by an equation of the form $f(x, y)=0$. Because the initial position $\left(x_{0}, y_{0}\right)$ and the final position $\left(x_{f}, y_{f}\right)$ of the finger were known, we defined the following two functions:

For the straight finger path case, we set

$$
f^{S}(x, y)=\left(y-y_{0}\right)-\frac{y_{f}-y_{0}}{x_{f}-x_{0}}\left(x-x_{0}\right)
$$

For the semicircular finger path case, we set

$$
f^{C}(x, y)=\left(x-x_{c}\right)^{2}+\left(y-y_{c}\right)^{2}-\left(\frac{\mathrm{LD}}{2}\right)^{2}
$$

and kept only the part of the curve in the semiplane defined by $f^{s}(x, y) \geq 0$. The latter equation described an arc of a circle. The point $\left(x_{c} y_{c}\right)$ was the middle of the segment of length LD that was defined in Materials and Methods.

The relationship between the elevation angles and the finger position was given by the following: 


$$
\left\{\begin{array}{c}
x=x_{\mathrm{Ak}}-l_{\mathrm{Sk}} \sin \theta_{\mathrm{Sk}}-l_{\mathrm{Th}} \sin \theta_{\mathrm{Th}} \\
-l_{\mathrm{Pe}} \sin \theta_{\mathrm{Pe}}-l_{\mathrm{Tr}} \sin \theta_{\mathrm{Tr}} \\
+l_{\mathrm{Hu}} \sin \theta_{\mathrm{Hu}}+l_{\mathrm{Fo}} \sin \theta_{\mathrm{Fo}} \\
+1_{\mathrm{Ha}} \sin \theta_{\mathrm{Ha}} \\
y=y_{\mathrm{Ak}}+l_{\mathrm{Sk}} \cos \theta_{\mathrm{Sk}}+l_{\mathrm{Th}} \cos \theta_{\mathrm{Th}} \\
+1_{\mathrm{Pe}} \cos \theta_{\mathrm{Pe}}+l_{\mathrm{Tr}} \cos \theta_{\mathrm{Tr}} \\
-1_{\mathrm{Hu}} \cos \theta_{\mathrm{Hu}}-l_{\mathrm{Fo}} \cos \theta_{\mathrm{Fo}} \\
-1_{\mathrm{Ha}} \cos \theta_{\mathrm{Ha}}
\end{array} .\right.
$$

The $l_{i}$ parameter corresponded to the segment length of each segment, and $\left(x_{\mathrm{Ak}}, y_{\mathrm{Ak}}\right)$ corresponded to the ankle marker position. Notice that Equation 2 did not depend on the head angle.

The optimal problem was to find a final configuration $\Theta^{f}$ minimizing the error between the imposed finger path [given by equations $f(x, y)=0$ ] and the path resulting from the choice of $\Theta^{f}$, given by Equation 2. Notice that the initial limb configuration was already known and fixed in this procedure.

For the imposed straight finger path, the cost under minimization was defined as the straightness of the finger trajectory, which was measured with the correlation coefficient of the finger coordinates $(x, y)$.

For the imposed semicircular finger path, the cost was defined as the IPC parameter that had to tend to 0.5 if the finger trajectory was a circular arc.

For both conditions, we also tested to directly minimize the root mean squared error between the simulated and the imposed finger path, and this did not change significantly the kinematic strategy found by the optimal process.

To conserve a degree of biological validity, the following nonlinear constraints were added to the optimal problem: (1) The A-P position of the CoM was forced to remain in a position within the points of support bounded by the heels and toes. This length was evaluated as the distance between the foot markers plus $6 \mathrm{~cm}$ in both forward and backward directions. (2) The final body configuration was requested to be compatible with the reach of the target (using the finger marker position) and such that biological articular limits were respected (using the biological ranges of joint angles).

This problem was solved numerically by using the optimization Matlab toolbox. A SQP (sequential quadratic programming) method was used ( fmincon Matlab function). The minimization of the above costs was subject to nonlinear inequalities (position of the CoM and articular limits) and equalities (position of the finger on the target) defined in a user-supplied function.

Basically, the procedure was initialized to the final limb configuration recorded during the basic motor task (Fig. 9a). We also initialized the process with 10 other admissible final limbs configurations selected randomly to limit the problem of local minima, and the resulting kinematic solutions were strongly similar whatever the chosen initial limb configuration. The solutions that we got are depicted in Figure $9 b-d$.

\section{References}

Alexandrov A, Frolov A, Massion J (1998) Axial synergies during human upper trunk bending. Exp Brain Res 118:210-220.

Alexandrov AV, Frolov AA, Massion J (2001a) Biomechanical analysis of movement strategies in human forward trunk bending. I. Modeling. Biol Cybern 84:425-434.

Alexandrov AV, Frolov AA, Massion J (2001b) Biomechanical analysis of movement strategies in human forward trunk bending. II. Experimental study. Biol Cybern 84:435-443.

Babinski J (1899) De l’asynergie cérébelleuse. Rev Neurol 7:806-816.

Belenkii V, Gurfinkel VS, Paltsev Y (1967) Elements of control of voluntary movements. Biofizika 12:135-141.

Bernstein N (1967) The coordination and regulation of movements. Oxford: Pergamon.

Bizzi E, Cheung VC, d'Avella A, Saltiel P, Tresch M (2008) Combining modules for movement. Brain Res Rev 57:125-133.

Capaday C (2004) The integrated nature of motor cortical function. Neuroscientist 10:207-220.

Cheron G, Bengoetxea A, Pozzo T, Bourgeois M, Draye JP (1997) Evidence of a preprogrammed deactivation of the hamstring muscles for triggering rapid changes of posture in humans. Electroencephalogr Clin Neurophysiol 105:58-71.

Cordo PJ, Nashner LM (1982) Properties of postural adjustments associated with rapid arm movements. J Neurophysiol 47:287-302.

Crenna P, Frigo C (1991) A motor programme for the initiation of forwardoriented movements in humans. J Physiol 437:635-653.

d'Avella A, Bizzi E (2005) Shared and specific muscle synergies in natural motor behaviors. Proc Natl Acad Sci U S A 102:3076-3081.

d'Avella A, Portone A, Fernandez L, Lacquaniti F (2006) Control of fastreaching movements by muscle synergy combinations. J Neurosci 26:7791-7810.

Dietz V (1992) Human neuronal control of automatic functional movements: interaction between central programs and afferent input. Physiol Rev 72:33-69.

Eng JJ, Winter DA, MacKinnon CD, Patla AE (1992) Interaction of the reactive moments and center of mass displacement for postural control during voluntary arm movements. Neurosci Res Commun 11:73-80.

Flash T, Hogan N (1985) The coordination of arm movements: an experimentally confirmed mathematical model. J Neurosci 5:1688-1703.

Hart CB, Giszter SF (2004) Modular premotor drives and unit bursts as primitives for frog motor behaviors. J Neurosci 24:5269-5282.

Hess WR (1954) Teleokinetisches und ereismatisches kräftesystem in der biomotorik. Helv Physiol Pharmacol Acta 1:C62-C63.

Horak F, McPherson J (1996) Postural orientation and equilibrium. In: Handbook of physiology (Rowell LB, Shepherd JT, eds). New York: Oxford UP.

Hufschmidt HJ, Hufschmidt T (1954) Antagonist inhibition as the earliest sign of a sensory-motor reaction. Nature 174:607.

Ivanenko YP, Cappellini G, Dominici N, Poppele RE, Lacquaniti F (2005) Coordination of locomotion with voluntary movements in humans. J Neurosci 25:7238-7253.

Jolliffe I (1986) Principal components analysis. New York: Springer.

Krouchev N, Kalaska JF, Drew T (2006) Sequential activation of muscle synergies during locomotion in the intact cat as revealed by cluster analysis and direct decomposition. J Neurophysiol 96:1991-2010.

Lacquaniti F, Grasso R, Zago M (1999) Motor patterns in walking. News Physiol Sci 14:168-174.

Massion J (1992) Movement, posture and equilibrium: interaction and coordination. Prog Neurobiol 38:35-56.

Mohan V, Morasso P (2006) A forward/inverse motor controller for cognitive robotics. Lect Notes Comput Sci 4131:602-611.

Mussa Ivaldi FA, Morasso P, Zaccaria R (1988) Kinematic networks. A distributed model for representing and regularizing motor redundancy. Biol Cybern 60:1-16.

Papaxanthis C, Pozzo T, McIntyre J (2005) Kinematic and dynamic processes for the control of pointing movements in humans revealed by short-term exposure to microgravity. Neuroscience 135:371-383.

Patron J, Stapley P, Pozzo T (2005) Human whole-body reaching in normal gravity and microgravity reveals a strong temporal coordination between postural and focal task components. Exp Brain Res 165:84-96.

Pigeon P, Bortolami SB, DiZio P, Lackner JR (2003) Coordinated turn-andreach movements. I. Anticipatory compensation for self-generated coriolis and interaction torques. J Neurophysiol 89:276-289.

Pozzo T, Berthoz A, Lefort L (1990) Head stabilization during various locomotor tasks in humans. I. Normal subjects. Exp Brain Res 82:97-106.

Pozzo T, Ouamer M, Gentil C (2001) Simulating mechanical consequences of voluntary movement upon whole-body equilibrium: the arm-raising paradigm revisited. Biol Cybern 85:39-49.

Pozzo T, Stapley PJ, Papaxanthis C (2002) Coordination between equilib- 
rium and hand trajectories during whole body pointing movements. Exp Brain Res 144:343-350.

Ramos CF, Stark LW (1990) Postural maintenance during fast forward bending: a model simulation experiment determines the "reduced trajectory." Exp Brain Res 82:651-657.

Schepens B, Drew T (2003) Strategies for the integration of posture and movement during reaching in the cat. J Neurophysiol 90:3066-3086.

Schepens B, Drew T (2004) Independent and convergent signals from the pontomedullary reticular formation contribute to the control of posture and movement during reaching in the cat. J Neurophysiol 92:2217-2238.

Schepens B, Stapley P, Drew T (2008) Neurons in the pontomedullary reticular formation signal posture and movement both as an integrated behavior and independently. J Neurophysiol 100:2235-2253.

Schmid M, Schieppati M, Pozzo T (2006) Effect of fatigue on the precision of a whole-body pointing task. Neuroscience 139:909-920.

Shadmehr R, Wise SP (2005) The computational neurobiology of reaching and pointing: a foundation for motor learning. Cambridge, MA: MIT.

Stapley P, Pozzo T, Grishin A (1998) The role of anticipatory postural adjustments during whole body forward reaching movements. Neuroreport 9:395-401.
Stapley PJ, Pozzo T, Cheron G, Grishin A (1999) Does the coordination between posture and movement during human whole-body reaching ensure center of mass stabilization? Exp Brain Res 129:134-146.

Thomas JS, Corcos DM, Hasan Z (2005) Kinematic and kinetic constraints on arm, trunk, and leg segments in target-reaching movements. J Neurophysiol 93:352-364.

Ting LH (2007) Dimensional reduction in sensorimotor systems: a framework for understanding muscle coordination of posture. Prog Brain Res 165:299-321.

Ting LH, Macpherson JM (2005) A limited set of muscle synergies for force control during a postural task. J Neurophysiol 93:609-613.

Viviani P, Flash T (1995) Minimum-jerk, two-thirds power law, and isochrony: converging approaches to movement planning. J Exp Psychol Hum Percept Perform 21:32-53.

Wada Y, Kaneko Y, Nakano E, Osu R, Kawato M (2001) Quantitative examinations for multi joint arm trajectory planning-using a robust calculation algorithm of the minimum commanded torque change trajectory. Neural Netw 14:381-393.

Winter D (1990) Biomechanics and motor control of human movement. New York: Wiley. 